\title{
Optical and IR observations of SN 2013L, a Type IIn Supernova surrounded by asymmetric CSM
}

\author{
Jennifer E. Andrews, ${ }^{1 \star}$ Nathan Smith, ${ }^{1}$ Curtis McCully, ${ }^{2,3}$ Ori D. Fox, ${ }^{4}$ \\ S. Valenti ${ }^{5}$ and D. A. Howell ${ }^{2,3}$ \\ ${ }^{1}$ Steward Observatory, University of Arizona, 933 North Cherry Avenue, Tucson, AZ 85721, USA \\ ${ }^{2}$ Las Cumbres Observatory, 6740 Cortona Dr Suite 102, Goleta, CA 93117, USA \\ ${ }^{3}$ Department of Physics, University of California, Santa Barbara, CA 93106, USA \\ ${ }^{4}$ Space Telescope Science Institute, 3700 San Martin Drive, Baltimore, MD 21218, USA \\ ${ }^{5}$ Department of Physics, University of California, Davis, 1 Shields Ave, Davis, CA 95616, USA
}

Accepted 2017 July 18. Received 2017 July 17; in original form 2017 June 9

\begin{abstract}
We present optical and near-IR photometry and spectroscopy of SN 2013L for the first $4 \mathrm{yr}$ post-explosion. SN 2013L was a moderately luminous $\left(M_{r}=-19.0\right)$ Type IIn supernova (SN) that showed signs of strong shock interaction with the circumstellar medium (CSM). The CSM interaction was equal to or stronger to SN $1988 \mathrm{Z}$ for the first $200 \mathrm{~d}$ and is observed at all epochs after explosion. Optical spectra revealed multicomponent hydrogen lines appearing by day 33 and persisting and slowly evolving over the next few years. By day 1509, the $\mathrm{H} \alpha$ emission was still strong and exhibiting multiple peaks, hinting that the CSM was in a disc or torus around the SN. SN 2013L is part of a growing subset of SNe IIn that shows both strong CSM interaction signatures and the underlying broad lines from the SN ejecta photosphere. The presence of a blue $\mathrm{H} \alpha$ emission bump and a lack of a red peak does not appear to be due to dust obscuration since an identical profile is seen in $\mathrm{Pa} \beta$. Instead this suggests a high concentration of material on the near-side of the SN or a disc inclination of roughly edge-on and hints that SN 2013L was part of a massive interactive binary system. Narrow $\mathrm{H} \alpha \mathrm{P}$-Cygni lines that persist through the entirety of the observations measure a progenitor outflow speed of $80-130 \mathrm{~km} \mathrm{~s}^{-1}$, speeds normally associated with extreme red supergiants, yellow hypergiants, or luminous blue variable winds. This progenitor scenario is also consistent with an inferred progenitor mass-loss rate of $0.3-8.0 \times 10^{-3} \mathrm{M}_{\odot} \mathrm{yr}^{-1}$.
\end{abstract}

Key words: circumstellar matter-supernovae: general-stars: winds, outflows.

\section{INTRODUCTION}

The Type II class of core collapse supernovae (CCSNe) come from massive stars $\left(>8 \mathrm{M}_{\odot}\right)$ that still contain at least some portion of their hydrogen envelope. Approximately 8-9 per cent of CCSNe show the presence of narrow $\left(\sim 100 \mathrm{~km} \mathrm{~s}^{-1}\right)$ hydrogen emission lines in their spectra (Smith et al. 2011b), due to the photoionization or shock heating of the surrounding, slow moving circumstellar medium (CSM). These CCSNe are classified as Type IIn (Schlegel 1990), where the 'n' stands for narrower lines than seen in normal Type II SNe.

Depending on the extent and density of the CSM, some SNe IIn show narrow lines only fleetingly (Gal-Yam et al. 2014; Smith et al. 2015) and others can exhibit additional broad $\left(\sim 10000 \mathrm{~km} \mathrm{~s}^{-1}\right)$ and intermediate $\left(\sim 1000 \mathrm{~km} \mathrm{~s}^{-1}\right)$ hydrogen

^E-mail: jandrews@as.arizona.edu emission lines for their full duration. The broad component traces emission from the free expansion of the $\mathrm{SN}$ ejecta, while the intermediate components are formed in the post-shock region between the forward and reverse shocks created as the ejecta moves through the CSM. This shock interaction transforms the kinetic energy of the ejecta into radiative energy, adding additional luminosity to the SN light curve. For some objects, this interaction can keep the late-time light curve bright for months or years after explosion (Aretxaga et al. 1999; Pastorello et al. 2002; Stritzinger et al. 2012; Fox et al. 2013, 2015; Smith et al. 2017). See Smith (2016) for a review of CSM interacting SNe.

While the presence of narrow emission lines is the unifying factor for these SNe, they are far from homogeneous. They can span absolute magnitudes between -22 and -17 , have progenitor mass-loss rates between $10^{-4}$ and $1 \mathrm{M}_{\odot} \mathrm{yr}^{-1}$ and wind velocities between 40 and $1000 \mathrm{~km} \mathrm{~s}^{-1}$ (Kiewe et al. 2012; Smith et al. 2007a, 2008b; Benetti et al. 1998; Taddia et al. 2013). These differences arise due to a variety of different progenitors and mass-loss histories (see 


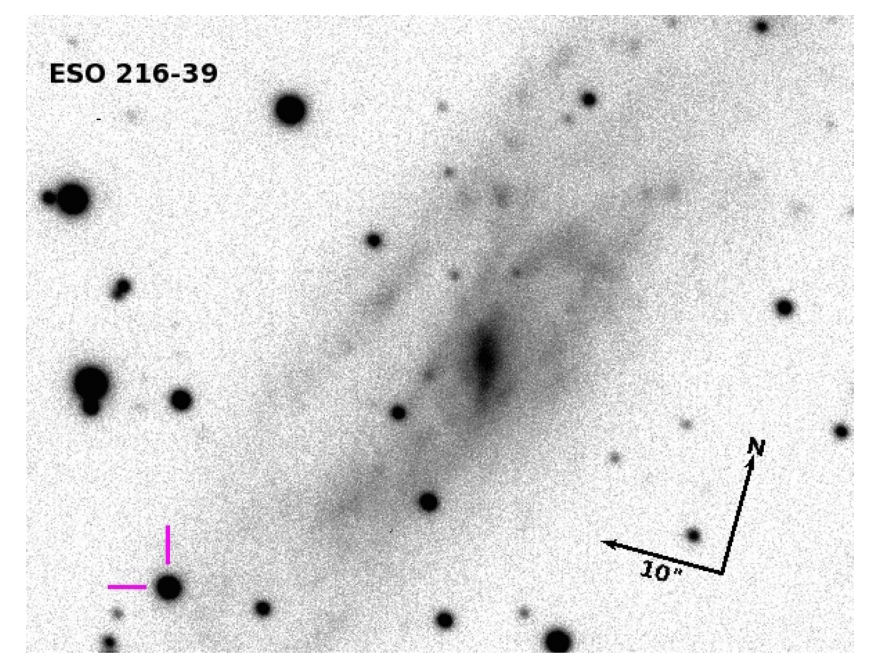

Figure 1. Magellan/IMACS $R$-band image of SN2013L taken on 2013 April 16 (day 84). The SN is indicated by the magenta cross hairs.

Smith 2016 for a full review). The progenitors for IIn showing signs of slower wind velocities $\left(40-100 \mathrm{~km} \mathrm{~s}^{-1}\right)$ and smaller mass-loss rates $\left(10^{-4}-10^{-3} \mathrm{M}_{\odot} \mathrm{yr}^{-1}\right)$ are likely extreme red supergiant (RSG) or yellow hypergiant (YHG) progenitors (Smith 2014). For instance the IIn SNe SN 2005ip (Smith et al. 2017), PTF11iqb (Smith et al. 2015), SN 2013fc (Kangas et al. 2016) and SN 1998S (Mauerhan \& Smith 2012) likely had RSG progenitors. Luminous blue variables (LBVs), traditionally not considered as a terminal point for massive star evolution, have been cited as progenitors of IIn that show signs of moderately fast winds $\left(\sim 100-600 \mathrm{~km} \mathrm{~s}^{-1}\right)$ and high mass-loss rates (Mauerhan et al. 2013b; Gal-Yam \& Leonard 2009; Smith et al. 2007a, 2008b, 2010, 2011a,c), but see Fox et al. (2017) for an alternative progenitor scenario of SN 2010j1. These progenitors have masses $>30 \mathrm{M}_{\odot}$, and sometime show signs of presupernova outbursts (Mauerhan et al. 2013a; Pastorello et al. 2013; Ofek et al. 2016), although not always (Bilinski et al. 2015). Interestingly, it appears that SNe IIn are not strongly correlated with $\mathrm{H} \alpha$ regions (Kangas et al. 2017; Habergham et al. 2014). This may hint that RSGs are the likely progenitors for all but the most luminous Type IIn (Smith, Hinkle \& Ryde 2009b).

To date, no definitive progenitor scenario seems to exist for these interacting $\mathrm{SNe}$, and it is likely that they can arise from a wide variety of progenitor masses. Additionally, it is important to remember that up to 75 per cent of massive stars are in binaries with separations close enough for interaction (Kiminki \& Kobulnicky 2012; Sana et al. 2012; de Mink et al. 2014); therefore, we should not neglect binary interaction as a potential channel for IIn production. Instabilities in a single massive progenitor and/or the presence of a companion can lead to sustained and often asymmetric mass-loss (Quataert \& Shiode 2012; Smith \& Arnett 2014). Whether from a single star or a binary system, when significant mass-loss events occur prior to core collapse, the result is narrow lines soon after explosion.

SN2013L $\quad\left[R A(2000)=11^{\mathrm{h}} 45^{\mathrm{m}} 29.55, \quad\right.$ Dec. $(2000)=$ $-50^{\circ} 35^{\prime} 53^{\prime \prime}$. 1] was discovered in a southeastern spiral arm of ESO 216-39 on 2013 January 22, and classified as a young Type IIn supernova shortly thereafter based on the presence of narrow Balmer emission lines (Monard et al. 2013, Fig. 1). The exact explosion date, while likely within a few days of the discovery, is unknown so in this paper all epochs will be relative to the date of discovery. Using emission lines in the $\mathrm{H}_{\text {II }}$ region associated with the $\mathrm{SN}$ we measure a redshift $z=0.01738$. Assuming $\mathrm{H}_{0}=$ $71.9 \mathrm{~km} \mathrm{~s}^{-1} \mathrm{Mpc}^{-1}$ (Bonvin et al. 2017), this gives an uncorrected rotational distance of $72 \mathrm{Mpc}$. The NASA/IPAC Extragalactic Databas $(\mathrm{NED})^{1}$ lists a Galactocentric distance of $67 \mathrm{Mpc}$, a distance of 73.4 corrected for the motion with respect to the $3 \mathrm{~K}$ CMB and a distance corrected for the Great Attractor and Virgo of $80.5 \mathrm{Mpc}$. Therefore, we adopt a distance of $72_{-5}^{+8.5} \mathrm{Mpc}$ throughout the paper. If the unfiltered discovery magnitude of 15.6 was peak brightness and if we adopt the Milky Way line-of-sight reddening of $E(B-V)=0.11 \mathrm{mag}$ (Schlegel, Finkbeiner \& Davis 1998), this gives an estimated absolute magnitude at peak of $-19.0 \pm 0.2$. Here, we present the optical photometric and spectroscopic evolution of SN 2013L from 5 to $1509 \mathrm{~d}$ after discovery. The data and their reduction will be discussed in Section 2, the photometric and spectroscopic evolution in Sections 3 and 4, the implications for the progenitor in Section 5, and finally a summary of conclusions will be presented in Section 6 .

\section{OBSERVATIONS}

\subsection{Imaging}

Starting on day 30 , optical monitoring in $g^{\prime} r^{\prime} i^{\prime} B V$ filters was conducted using the Las Cumbres Observatory (LCO) network. ${ }^{2}$ These observations constitute our early light-curve data, seen in Fig. 2. The images were reduced using the lcogtsnpipe pipeline (Valenti et al. 2016), and photometric calibration was done using APASS $^{3}$ standards.

We also obtained 20-s $R$-band exposures with Magellan/IMACS (Dressler et al. 2011) on days 84, 482, 1133 and 1509 after explosion. On day 728, 60-s exposures in each $V R i^{\prime}$ filter were taken. Standard reduction techniques with ira $^{4}$ were used, and absolute calibration was performed using APASS standards and aperture photometry. Transformation between $r^{\prime}$ and $R$ for the standards was carried out using Jester et al. (2005), and tertiary standards were created across all IMACS images to create a robust zero-point. The photometry is summarized in Table 1.

Spitzer IRAC (3.6 and $4.5 \mu \mathrm{m}$ ) images on days 630 and 850 were retrieved from the Spitzer Heritage Archive as part of an ongoing programme by PI Fox (PID 10139, 11053). Aperture photometry was performed on the pipeline reduced pbcd images, with the appropriate aperture corrections. A summary of dates and fluxes are shown in Table 2, and an example of the images can be seen in Fig. 3.

\subsection{Optical spectra}

We obtained 1 epoch of low resolution and 4 epochs of moderateresolution optical long-slit spectra with IMACS on Magellan/Baade on 2013 April 17, 2014 May 19, 2015 January 1, 2016 March 4 and 2017 March 11 (see Table 3). The first observation $84 \mathrm{~d}$ after explosion was taken with the $3001 \mathrm{~mm}^{-1}$ grating and $3 \times 120 \mathrm{~s}$

\footnotetext{
${ }^{1}$ The NED is operated by the Jet Propulsion Laboratory, California Institute of Technology, under contract with the National Aeronautics and Space Administration.

${ }^{2}$ http://lcogt.net/

${ }^{3}$ https://www.aavso.org/apass

${ }^{4}$ IRAF, the Image Reduction and Analysis Facility, is distributed by the National Optical Astronomy Observatory, which is operated by the Association of Universities for Research in Astronomy under cooperative agreement with the National Science Foundation (NSF).
} 


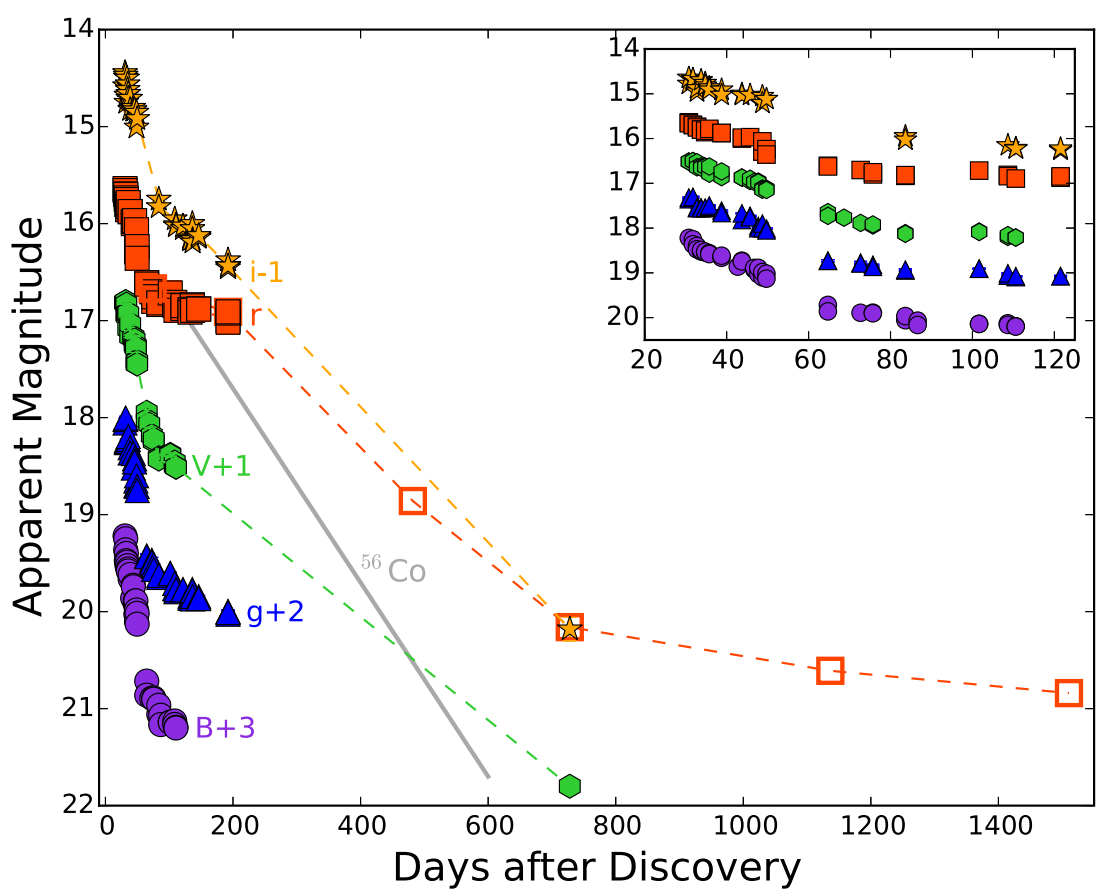

Figure 2. Light curve of SN2013L in multiple bands. Filters have been shifted for clarity and are indicated on the plot. The inset shows a blow-up of the first $120 \mathrm{~d}$, and the grey solid line indicates the radioactive decay of ${ }^{56} \mathrm{Co} . R$-band Magellan/IMACS photometry is indicated by the open squares. The photometry have not been corrected for extinction and come directly from the values listed in Table 1.

exposures, while the later epochs used the $12001 \mathrm{~mm}^{-1}$ grating centred on $\mathrm{H} \alpha$ and $3 \times 400$ s exposures. Table 3 reports the slit width used for each observation, as well as resolving power. Standard reductions were carried out using IRAF on a chip-by-chip basis. Flux calibration was achieved using spectrophotometric standards at a similar airmass taken with each science frame.

We have also obtained the discovery spectrum cited in Morales Garoffolo et al. (2013) taken with ESO New Technology Telescope at La Silla on 2013 January 27, using EFOSC2 and made available via the PESSTO $^{5}$ campaign (Smartt et al. 2015), and found on the WISeRep ${ }^{6}$ archive (Yaron \& Gal-Yam 2012). Additionally, we have included VLT/XSHOOTER spectroscopy from GTO Program 090.D-0719(A), PI Hjorth made available as a Phase 3 data product on the ESO archive. Full-spectral evolution of all epochs are shown in Fig. 4.

\section{PHOTOMETRIC EVOLUTION}

\subsection{Optical light curve}

Fig. 2 presents the full photometric evolution of SN 2013L in $g^{\prime} r^{\prime} i^{\prime} B V$, with the first $120 \mathrm{~d}$ shown in detail in the inset. The light curves are reminiscent of Type IIL supernovae, with the first $50 \mathrm{~d}$ or so of photometric evolution in all filters being approximately linear, at a decline rate of $0.03 \mathrm{mag} \mathrm{d}^{-1}$ in $r^{\prime}$ and $V$. Around day 60, $r^{\prime}$ dramatically slows to a decline rate of $0.003 \mathrm{mag} \mathrm{d}^{-1}$ that lasts for the next $140 \mathrm{~d}$, until we lose the SN behind the Sun. During the same time period, the other filters slow their decline rate as well, but are not as flat as $r^{\prime}$, which includes the $\mathrm{H} \alpha$ emission produced by CSM interaction. After day 200, the sparse observations indicate a

\footnotetext{
${ }^{5}$ Public ESO Spectroscopic Survey of Transient Objects (www.pessto.org).

${ }^{6} \mathrm{http}: / /$ wiserep.weizmann.ac.il/
}

steady drop of around 3 mag over a $500 \mathrm{~d}$ period, which then slows

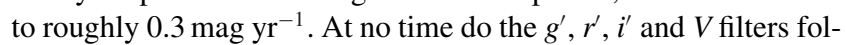
low the radioactive ${ }^{56} \mathrm{Co}$ decay rate of $\sim 0.01 \mathrm{mag} \mathrm{d}^{-1}$. This requires additional luminosity from late-time CSM interaction.

In Fig. 5, we illustrate the distinctive optical evolution of SN 2013L by comparing the absolute $r^{\prime} / R$ light curve with those of other interacting SNe. At early times, SN 2013L most resembles SN 1998S (a IIn SNe with IIL-type light curve) and has a significantly brighter absolute magnitude than the IIn SN2005ip and IIP SN2004dj. From days 60-90 post-discovery, the magnitude and decline rate of SN 2013L mirrors SN 1988Z, PTF11iqb and SN 2007 od, the latter of which is a 'normal' SN IIP. After day 100, the light curves diverge as the shock interaction begins to take over as the main energy source of SN 2013L.

Between 100 and $200 \mathrm{~d}$, the absolute magnitude of SN 2013L stays abnormally bright at around $-17.7 \mathrm{mag}$, comparable only with the enduring IIn SN 1988Z that is still showing signs of interaction today (Smith et al. 2017). Only after day 200 does the luminosity begin to fade below that of SN $1988 \mathrm{Z}$ and by $2 \mathrm{yr}$ post-explosion, the absolute $R$ magnitude of SN 2013L resides between that of SN 2005ip and PTF11iqb (a similar pattern in $\mathrm{H} \alpha$ is observed and discussed below). At all times, the light curve of SN 2004dj, a Type IIP SN with circumstellar interaction, is significantly fainter than the other SNe due to the lower density CSM surrounding a IIP explosion.

\subsection{Colour evolution}

A look at the colour evolution of SN 2013L further highlights the similarities with both SN 1998 S and PTF11iqb (Fig. 6). While the photometric coverage is sparse prior to day 35 , an extrapolation suggests the colour started quite blue, likely $B-V \sim-0.1$ on day 5 , using the blackbody temperature of $12000 \mathrm{~K}$ and the method presented in Ballesteros (2012). The colour then gradually evolved 
Table 1. Optical photometry of SN 2013L obtained with LCO and Magellan/IMACS*.

\begin{tabular}{|c|c|c|c|c|c|}
\hline JD & $g$ & $r$ & $i$ & $B$ & $V$ \\
\hline 2456344.75 & $16.0559 \pm 0.0154$ & $15.6389 \pm 0.0143$ & $15.5815 \pm 0.0171$ & $16.2195 \pm 0.0145$ & $15.8145 \pm 0.0171$ \\
\hline 2456344.76 & $16.0188 \pm 0.014$ & $15.6595 \pm 0.0249$ & $15.4453 \pm 0.0134$ & $\ldots$ & $15.8149 \pm 0.0163$ \\
\hline 2456345.76 & $16.0206 \pm 0.0182$ & $15.6941 \pm 0.0141$ & $15.4923 \pm 0.0133$ & $16.2489 \pm 0.0169$ & $15.8051 \pm 0.0156$ \\
\hline 2456345.76 & $16.0042 \pm 0.0184$ & $15.6985 \pm 0.0156$ & $15.4714 \pm 0.015$ & $16.3627 \pm 0.0188$ & $15.8028 \pm 0.019$ \\
\hline 2456346.76 & $16.2382 \pm 0.0173$ & $15.7351 \pm 0.0185$ & $15.7514 \pm 0.0222$ & $16.4003 \pm 0.0319$ & $15.8293 \pm 0.0263$ \\
\hline 2456346.76 & $16.2556 \pm 0.0128$ & $15.7521 \pm 0.0169$ & $15.6777 \pm 0.0231$ & $16.4748 \pm 0.0229$ & $15.9417 \pm 0.0198$ \\
\hline 2456347.76 & $16.2368 \pm 0.0161$ & $15.8027 \pm 0.0173$ & $15.5017 \pm 0.0136$ & $16.5229 \pm 0.0166$ & $15.9215 \pm 0.0151$ \\
\hline 2456347.76 & $16.2586 \pm 0.0164$ & $15.8003 \pm 0.0171$ & $\ldots$ & $16.4904 \pm 0.0163$ & $15.952 \pm 0.0205$ \\
\hline 2456348.76 & $16.2554 \pm 0.0144$ & $15.8567 \pm 0.0154$ & $15.5615 \pm 0.02$ & $16.5445 \pm 0.019$ & $15.9284 \pm 0.0168$ \\
\hline 2456348.76 & $16.2462 \pm 0.0128$ & $15.824 \pm 0.0173$ & $15.619 \pm 0.0154$ & $16.5261 \pm 0.0193$ & $15.933 \pm 0.0159$ \\
\hline 2456349.76 & $16.2256 \pm 0.0175$ & $15.8268 \pm 0.0162$ & $15.6599 \pm 0.02$ & $16.56 \pm 0.0241$ & $16.0859 \pm 0.0144$ \\
\hline 2456349.76 & $16.1953 \pm 0.016$ & $15.7834 \pm 0.0211$ & $15.7098 \pm 0.0209$ & $16.5809 \pm 0.0216$ & $15.9249 \pm 0.0152$ \\
\hline 2456352.76 & $16.3108 \pm 0.0174$ & $15.8777 \pm 0.0145$ & $15.7102 \pm 0.0163$ & $16.6634 \pm 0.013$ & $16.162 \pm 0.0129$ \\
\hline 2456352.76 & $16.3647 \pm 0.0145$ & $15.8758 \pm 0.0115$ & $15.8261 \pm 0.02$ & $16.6168 \pm 0.0131$ & $16.035 \pm 0.0129$ \\
\hline 2456356.71 & $\cdots$ & $\cdots$ & $\cdots$ & $16.8604 \pm 0.0281$ & $\cdots$ \\
\hline 2456357.72 & $16.5205 \pm 0.0108$ & $16.0003 \pm 0.0116$ & $15.8035 \pm 0.0116$ & $16.728 \pm 0.0171$ & $16.1711 \pm 0.0158$ \\
\hline 2456357.72 & $16.3707 \pm 0.0115$ & $15.9794 \pm 0.0108$ & $15.8334 \pm 0.0107$ & $16.7437 \pm 0.0174$ & $16.1724 \pm 0.0121$ \\
\hline 2456359.72 & $16.4253 \pm 0.0122$ & $15.9693 \pm 0.0196$ & $15.8283 \pm 0.0512$ & $16.9236 \pm$ & $16.2479 \pm 0.0805$ \\
\hline 2456359.72 & $16.4569 \pm 0.0163$ & $\ldots$ & $\ldots$ & $\ldots$ & $16.1982 \pm 0.0359$ \\
\hline 2456360.71 & $\ldots$ & $\cdots$ & $\cdots$ & $16.9236 \pm 0.0167$ & $16.2689 \pm 0.0214$ \\
\hline 2456360.71 & $\ldots$ & $\cdots$ & $\cdots$ & $16.8899 \pm 0.0216$ & $16.261 \pm 0.0177$ \\
\hline 2456361.72 & $16.6534 \pm 0.0268$ & $\cdots$ & $\cdots$ & $17.0263 \pm 0.0158$ & $16.2801 \pm 0.0194$ \\
\hline 2456361.73 & $16.7027 \pm 0.0214$ & $\ldots$ & $\ldots$ & $16.8886 \pm 0.0181$ & $\ldots$ \\
\hline 2456362.72 & $16.7052 \pm 0.0153$ & $16.2899 \pm 0.0136$ & $15.8535 \pm 0.0102$ & $17.0989 \pm 0.018$ & $16.4172 \pm 0.0153$ \\
\hline 2456362.72 & $16.5996 \pm 0.0156$ & $16.0636 \pm 0.0163$ & $16.0068 \pm 0.0248$ & $16.9833 \pm 0.0156$ & $16.4432 \pm 0.0145$ \\
\hline 2456363.72 & $16.7105 \pm 0.0177$ & $16.2372 \pm 0.0196$ & $15.919 \pm 0.03$ & $17.0173 \pm 0.025$ & $16.4207 \pm 0.0225$ \\
\hline 2456363.72 & $16.7522 \pm 0.0254$ & $16.3574 \pm 0.0241$ & $\ldots$ & $17.1292 \pm 0.0229$ & $16.449 \pm 0.0215$ \\
\hline 2456378.72 & $17.4258 \pm 0.021$ & $16.6082 \pm 0.0245$ & $\cdots$ & $17.716 \pm 0.0311$ & $16.9443 \pm 0.0287$ \\
\hline 2456378.72 & $17.4339 \pm 0.0278$ & $16.6235 \pm 0.0219$ & $\cdots$ & $17.8611 \pm 0.0371$ & $17.0272 \pm 0.034$ \\
\hline 2456382.65 & $\cdots$ & $\cdots$ & $\cdots$ & $\cdots$ & $17.0673 \pm 0.0322$ \\
\hline 2456386.70 & $17.471 \pm 0.0305$ & $16.7031 \pm 0.0296$ & $\cdots$ & $17.8922 \pm 0.0193$ & $17.1843 \pm 0.0172$ \\
\hline 2456386.70 & $17.4914 \pm 0.0108$ & $\cdots$ & $\cdots$ & $\cdots$ & $\cdots$ \\
\hline $2456388.50 *$ & $\cdots$ & $16.65 \pm 0.1$ & $\cdots$ & $\ldots$ & $\ldots$ \\
\hline 2456389.70 & $17.5188 \pm 0.0142$ & $16.8021 \pm 0.0116$ & $\cdots$ & $17.8898 \pm 0.0169$ & $17.2418 \pm 0.0155$ \\
\hline 2456389.70 & $17.5657 \pm 0.0151$ & $16.7536 \pm 0.0124$ & $\ldots$ & $17.9031 \pm 0.0154$ & $17.2194 \pm 0.0199$ \\
\hline 2456397.63 & $17.6481 \pm 0.0207$ & $16.8336 \pm 0.0255$ & $16.7504 \pm 0.0245$ & $18.0585 \pm 0.0269$ & $17.4132 \pm 0.0388$ \\
\hline 2456397.63 & $17.6267 \pm 0.022$ & $16.8172 \pm 0.0281$ & $16.8276 \pm 0.0158$ & $17.9653 \pm 0.0198$ & $17.4284 \pm 0.0368$ \\
\hline 2456400.62 & $\ldots$ & $\ldots$ & $\cdots$ & $18.068 \pm 0.0254$ & $\ldots$ \\
\hline 2456400.62 & $\cdots$ & $\cdots$ & $\cdots$ & $18.1644 \pm 0.0285$ & $\cdots$ \\
\hline 2456415.64 & $17.6046 \pm 0.0115$ & $16.7153 \pm 0.0115$ & $\ldots$ & $18.1384 \pm 0.0156$ & $17.3786 \pm 0.0165$ \\
\hline 2456415.64 & $17.6094 \pm 0.0117$ & $\cdots$ & $\cdots$ & $18.1416 \pm 0.0191$ & $17.387 \pm 0.0148$ \\
\hline 2456422.65 & $17.7628 \pm 0.0122$ & $16.8116 \pm 0.0101$ & $16.9529 \pm 0.0147$ & $18.1274 \pm 0.0188$ & $17.4978 \pm 0.0148$ \\
\hline 2456422.65 & $17.7101 \pm 0.0104$ & $16.841 \pm 0.01$ & $\cdots$ & $18.1631 \pm 0.0172$ & $17.4575 \pm 0.0156$ \\
\hline 2456424.58 & $17.7955 \pm 0.0154$ & $16.8936 \pm 0.0147$ & $17.0141 \pm 0.0214$ & $18.1917 \pm 0.0147$ & $17.5109 \pm 0.0183$ \\
\hline 2456424.58 & $17.7745 \pm 0.0124$ & $\ldots$ & $17.0255 \pm 0.0205$ & $18.1998 \pm 0.0149$ & $17.5116 \pm 0.0147$ \\
\hline 2456435.50 & $17.7734 \pm 0.0168$ & $16.8754 \pm 0.0156$ & $17.0539 \pm 0.0176$ & $\cdots$ & $\cdots$ \\
\hline 2456435.51 & $17.7757 \pm 0.0197$ & $16.8456 \pm 0.0138$ & $17.0262 \pm 0.0147$ & $\cdots$ & $\cdots$ \\
\hline 2456446.57 & $17.8406 \pm 0.0179$ & $16.8872 \pm 0.0166$ & $17.1504 \pm 0.0195$ & $\cdots$ & $\cdots$ \\
\hline 2456446.58 & $17.8631 \pm 0.018$ & $16.913 \pm 0.0148$ & $17.1702 \pm 0.0205$ & $\ldots$ & $\cdots$ \\
\hline 2456450.25 & $17.7719 \pm 0.0165$ & $16.8362 \pm 0.0118$ & $17.0005 \pm 0.0219$ & $\cdots$ & $\cdots$ \\
\hline 2456450.25 & $17.7858 \pm 0.0127$ & $16.8474 \pm 0.011$ & $17.053 \pm 0.0153$ & $\cdots$ & $\cdots$ \\
\hline 2456450.57 & $17.8697 \pm 0.016$ & $16.8832 \pm 0.0148$ & $17.1661 \pm 0.02$ & $\cdots$ & $\cdots$ \\
\hline 2456450.58 & $17.8438 \pm 0.0144$ & $16.8946 \pm 0.0149$ & $17.1921 \pm 0.0219$ & $\cdots$ & $\cdots$ \\
\hline 2456460.19 & $17.8451 \pm 0.0125$ & $16.878 \pm 0.0153$ & $17.1444 \pm 0.018$ & $\cdots$ & $\cdots$ \\
\hline 2456460.20 & $17.8663 \pm 0.0124$ & $16.8816 \pm 0.0132$ & $17.1245 \pm 0.0286$ & $\cdots$ & $\cdots$ \\
\hline 2456506.21 & $18.0043 \pm 0.0236$ & $16.9962 \pm 0.0146$ & $17.4327 \pm 0.0223$ & $\cdots$ & $\cdots$ \\
\hline 2456506.21 & $18.0269 \pm 0.0151$ & $17.0162 \pm 0.0099$ & $17.4566 \pm 0.0273$ & $\cdots$ & $\cdots$ \\
\hline 2456506.22 & $17.9992 \pm 0.0171$ & $16.9226 \pm 0.0144$ & $17.3753 \pm 0.0233$ & $\cdots$ & $\cdots$ \\
\hline 2456506.22 & $18.0095 \pm 0.0158$ & $16.9099 \pm 0.014$ & $17.4377 \pm 0.0204$ & $\cdots$ & $\cdots$ \\
\hline $2456796.62 *$ & $\cdots$ & $18.86 \pm 0.2$ & $\ldots$ & $\ldots$ & $\ldots$ \\
\hline $2457042.35 *$ & $\cdots$ & $20.16 \pm 0.1$ & $21.18 \pm 0.12$ & $\cdots$ & $20.8 \pm 0.06$ \\
\hline $2457450.50 *$ & $\cdots$ & $20.61 \pm 0.1$ & $\cdots$ & $\cdots$ & $\ldots$ \\
\hline $2457824.60 *$ & $\cdots$ & $20.84 \pm 0.1$ & $\cdots$ & $\ldots$ & $\ldots$ \\
\hline
\end{tabular}

*R mags. 
Table 2. Spitzer observations.

\begin{tabular}{lccc}
\hline Date & $\begin{array}{c}\text { Day } \\
(\text { discovery })\end{array}$ & $\begin{array}{c}3.6 \mu \mathrm{m} \\
(\mu \mathrm{Jy})\end{array}$ & $\begin{array}{c}4.5 \mu \mathrm{m} \\
(\mu \mathrm{Jy})\end{array}$ \\
\hline $2014-10-14$ & 630 & $742 \pm 41$ & $812 \pm 42$ \\
$2015-05-22$ & 850 & $677 \pm 40$ & $830 \pm 43$ \\
\hline
\end{tabular}

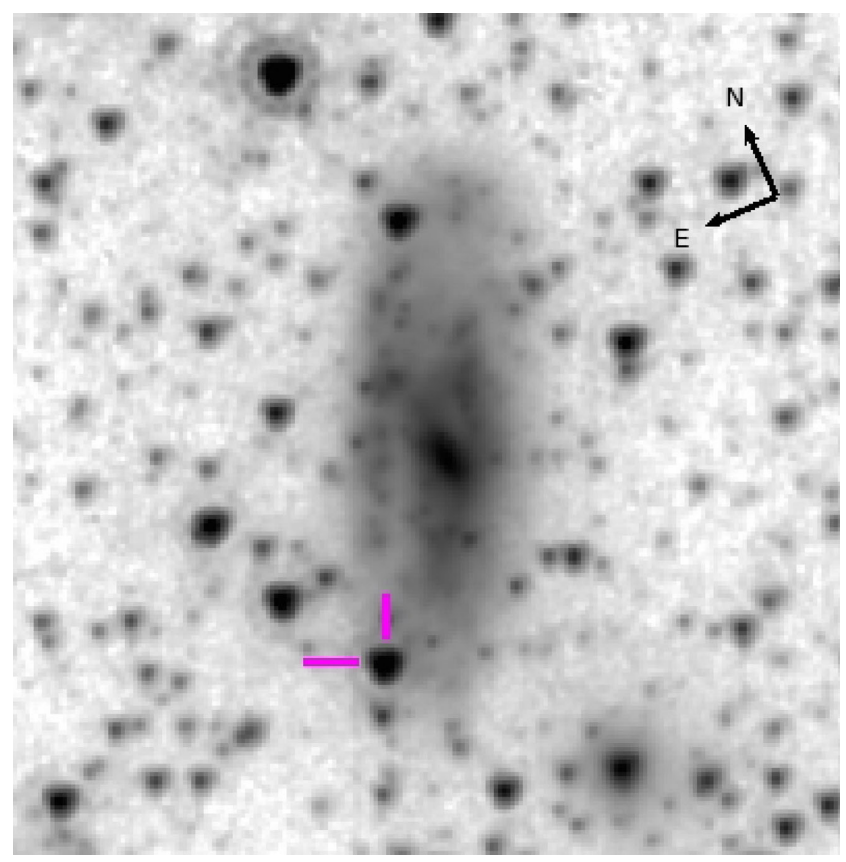

Figure 3. Spitzer $4.5-\mu \mathrm{m}$ image of SN2013L on day 850 . The $\mathrm{SN}$ is indicated by magenta cross hairs.

redward before levelling off at a value of $B-V=0.7$, a trend identical to the aforementioned objects. What is intriguing is that the spectra of SN 2013L show persistent narrow lines, indicative of normal and long-lasting IIn SNe, while the other two only briefly show them, yet all three exhibit the same colour evolution. Of course, one tie that does seem to bind these three objects together is the presence of asymmetric emission line profiles in the intermediate width and broad components of $\mathrm{H}$ lines, which we will discuss in detail in the next section.

The other two interacting Type IIP SNe shown for comparison, SNe 2007od and 2004dj, also start out quite blue but $B-V$ increases to $>1.2$ rather quickly. This pattern is the norm for most Type IIP $\mathrm{SNe}$, and as the CSM interaction of these objects is delayed, is not surprising. SN 2005ip on the other hand stays consistently around $B-V=0.4 \mathrm{mag}$, a pattern also seen in SN 1988Z and SN 2010j1 (Turatto et al. 1993; Zhang et al. 2012). Considering the late-time endurance of the light curve of SN 2013L, one could expect the colour evolution to mimic these objects with very strong CSM interaction and slow fading, yet it is redder and likely evolved that way rather quickly.

A quick examination of Fig. 6 shows that we could roughly subdivide these Type II events into three classes: the red Type IIP, the blue and flat IIn, and the intermediate 98S-like. The normal IIP supernovae quickly go from blue to red, as the large RSG envelope expands and cools. The classic IIn like SN2005ip on the other hand stay rather blue as the shock interaction keeps the temperature high, and often a blue pseudo-continuum, caused by a blend of narrow fluorescing metal lines in the surrounding CSM, can appear (Smith
Table 3. Optical spectroscopy of SN 2013L.

\begin{tabular}{lcccc}
\hline $\begin{array}{l}\text { Date } \\
(\mathrm{y}-\mathrm{m}-\mathrm{d})\end{array}$ & $\begin{array}{c}\text { Telescope } \\
(\text { Instrument })\end{array}$ & Day & $\begin{array}{c}\text { Slit width } \\
(\operatorname{arcsec})\end{array}$ & $\begin{array}{c}\mathrm{R} \\
(\lambda / \delta \lambda)\end{array}$ \\
\hline 2013-01-27 & NTT/EFOSC2 & 5 & 1.0 & 300 \\
$2013-02-24$ & VLT/XSHOOTER & 33 & 0.9 & 7400 \\
$2013-03-11$ & VLT/XSHOOTER & 48 & 0.9 & 7400 \\
$2013-03-31$ & VLT/XSHOOTER & 68 & 0.9 & 7400 \\
$2013-04-16$ & Magellan/IMACS & 84 & 0.7 & 1400 \\
$2013-05-11$ & VLT/XSHOOTER & 109 & 0.9 & 7400 \\
$2014-05-19$ & Magellan/IMACS & 482 & 0.7 & 5300 \\
$2015-01-20$ & Magellan/IMACS & 728 & 0.7 & 5300 \\
$2016-03-05$ & Magellan/IMACS & 1133 & 0.7 & 5300 \\
$2017-03-11$ & Magellan/IMACS & 1509 & 0.7 & 5300 \\
\hline
\end{tabular}

et al. 2009a). This would suggest that the intermediate IIn objects show a mix of the SN photosphere and CSM interaction, which likely is caused by asymmetry in the CSM. The spectral evolution of all these objects (SN 1998S, PTF11iqb, and SN 2013L) in factshow spectral signatures of an asymmetric CSM, as will be discussed more below.

\subsection{Infrared evolution}

Spitzer IRAC 3.6 and $4.5 \mu \mathrm{m}$ imaging from days 630 and 850 (Fig. 3) show a bright source at the location of SN 2013L. As we show in Table 2 the SN emission is roughly $0.8 \mathrm{mJy}$, relatively bright for an SN so distant and of that age. For comparison the $3.6 \mu \mathrm{m}$ flux for SN 2010jl on day 621 was $8.63 \mathrm{mJy}$ (Fransson et al. 2014) and for SN 2005ip on day 948 was $5.76 \mathrm{mJy}$ (Fox et al. 2011). If moved to the distance of SN 2013L (72 Mpc), these would correspond to 4.0 and $1.4 \mathrm{mJy}$, respectively. Both IIn SNe have components of pre-existing and newly formed dust (Fox et al. 2009; Smith et al. 2009a; Fox et al. 2010; Andrews et al. 2011; Smith et al. 2012; Gall et al. 2014), which could also be the case for SN 2013L. Whether this emission is from an IR echo, shock-heated pre-existing dust, or from newly formed dust grains can be difficult to disentangle. Unfortunately, no earlier Spitzer observations exist, so it is impossible to compare how the IR emission has evolved with time. We also cannot discount a nearby or underlying source as the cause of some or all of the emission, as no pre-SN imaging exists. This is unlikely because of the high luminosity $\left(>2.5 \times 10^{7} \mathrm{~L}_{\odot}\right)$ and a change in flux between the two observations presented here. Continued monitoring with Spitzer (and eventually James Webb Space Telescope) will allow us to decipher more precisely the IR environment surrounding SN 2013L.

A blackbody fit to the two Spitzer passbands can give us a rough idea of the temperature and location of the dust. Fig. 7 shows the best fits to the Spitzer epochs on days 630 and 850 . Both dates can be fit with temperatures of 1000 and $890 \mathrm{~K}$, respectively, a reasonable temperature for all three scenarios. The blackbody radius $\left(R_{\mathrm{bb}}\right)$ can give us a stringent lower limit of the location of the IR luminosity. $R_{\mathrm{bb}}$ grows from 1.5 to $1.8 \times 10^{16} \mathrm{~cm}$ between the two dates, or from 5 to 7 light days. By day 630, the initial flash of the SN has travelled well beyond 1.7 light years, but this does not necessarily discount the presence of a light echo scattering off of intervening gas and dust. It does however take a velocity of 2750 and $2450 \mathrm{~km} \mathrm{~s}^{-1}$ to travel the distance of $R_{\mathrm{bb}}$ in 630 and $850 \mathrm{~d}$, respectively. These velocities are almost identical to the central velocity of the bluecomponent seen in our $\mathrm{H} \alpha$ line around the same time (Fig. 8 and Table 4). This strongly advocates for pre-existing CSM that is being shock heated by the expanding SN ejecta. 

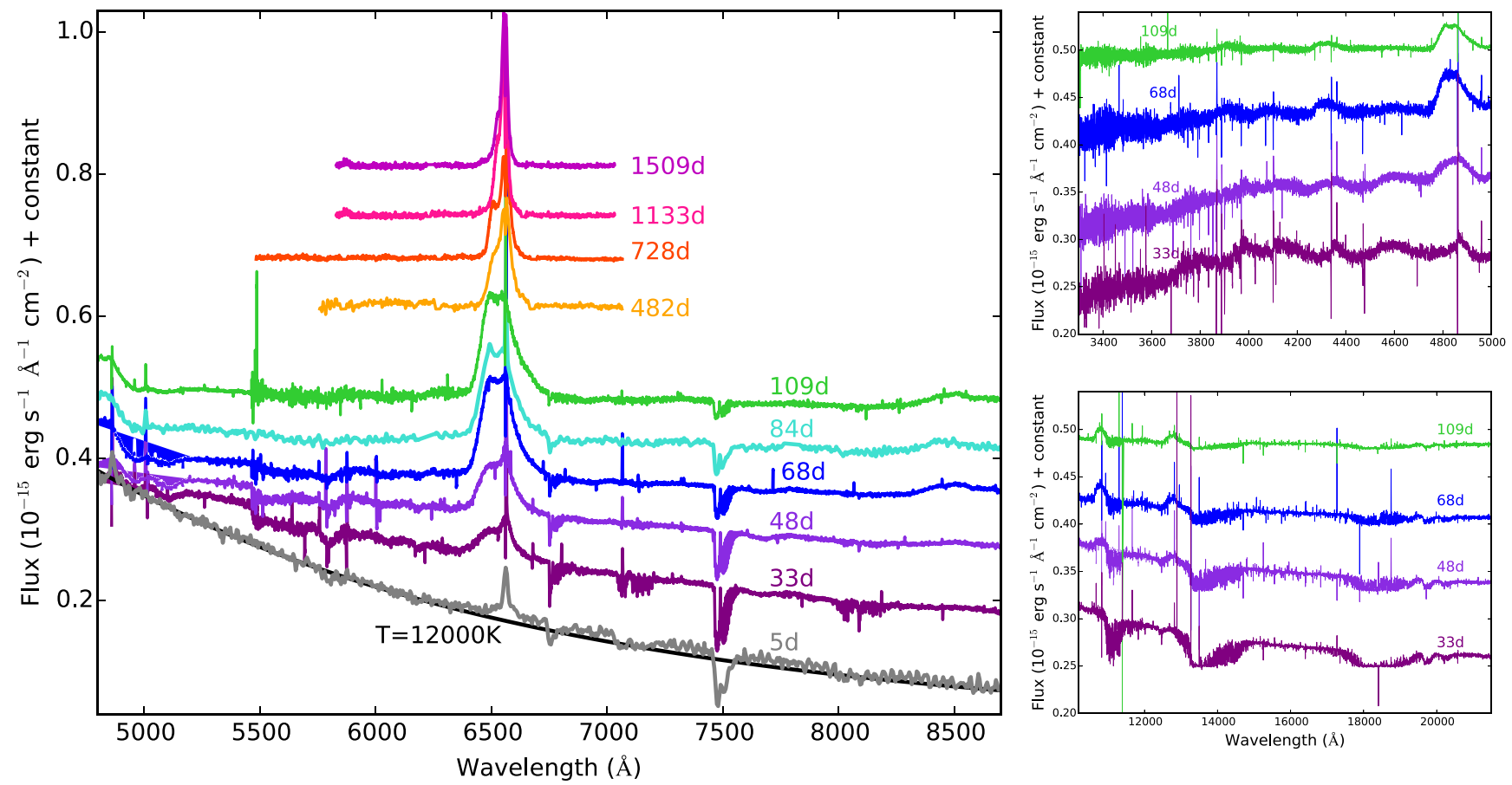

Figure 4. Spectroscopic evolution of SN 2013L from 5 d to $1509 \mathrm{~d}$ after discovery. A summary of observational parameters is given in Table 3. All spectra have been corrected for Milky Way reddening of $E(B-V)=0.11$ and have been shifted into the rest frame and are plotted in flux units plus a constant. A $12000 \mathrm{~K}$ blackbody has been plotted in black under the discovery spectrum for comparison. The XSHOOTER NUV and NIR data are plotted on the right.

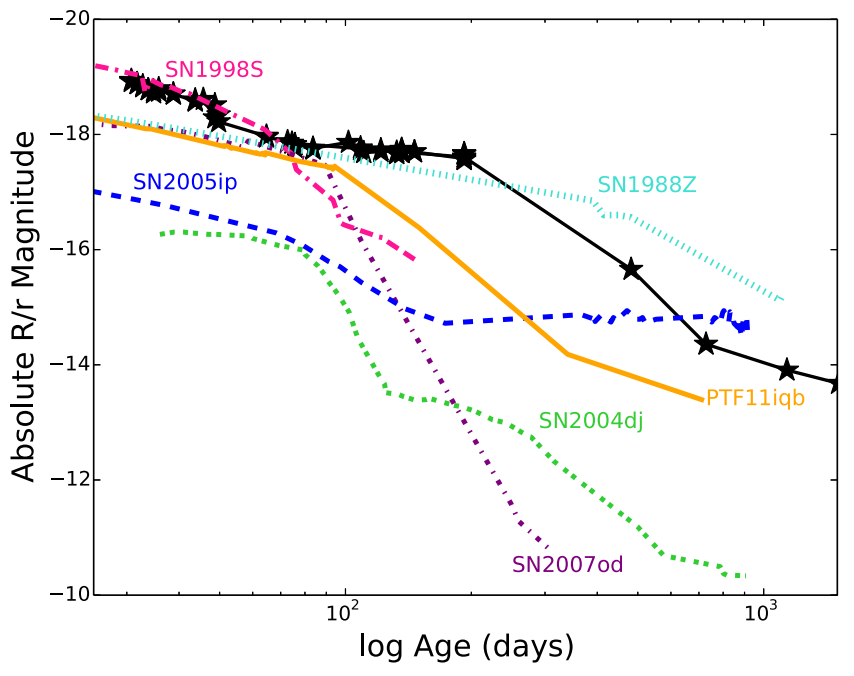

Figure 5. Absolute $R$ and $r^{\prime}$ light curves of CSM interacting CCSNe compared with SN 2013L (black stars). Data are from Smith et al. (2009a, SN2005ip), Smith et al. (2015, PTF1liqb), Fassia et al. (2000, SN1998S), Zhang et al. (2006, SN2004dj), Andrews et al. (2010, SN2007od) and Turatto et al. (1993, SN1988Z). All data have been corrected for distance and extinction using the $E(B-V)$ quoted in the corresponding papers, and using a standard reddening law of $R_{v}$ (Cardelli, Clayton \& Mathis 1989).

The dates, and more importantly, the temperatures of the IR observations are conducive to the formation of dust grains so we also cannot completely rule out new dust formation in the ejecta or post-shock gas. As we will discuss further in Section 4, we do believe the case for only newly formed dust is a bit weak, as the emission line asymmetries are most likely caused by the SN geometry rather than just dust attenuating the receding emission.

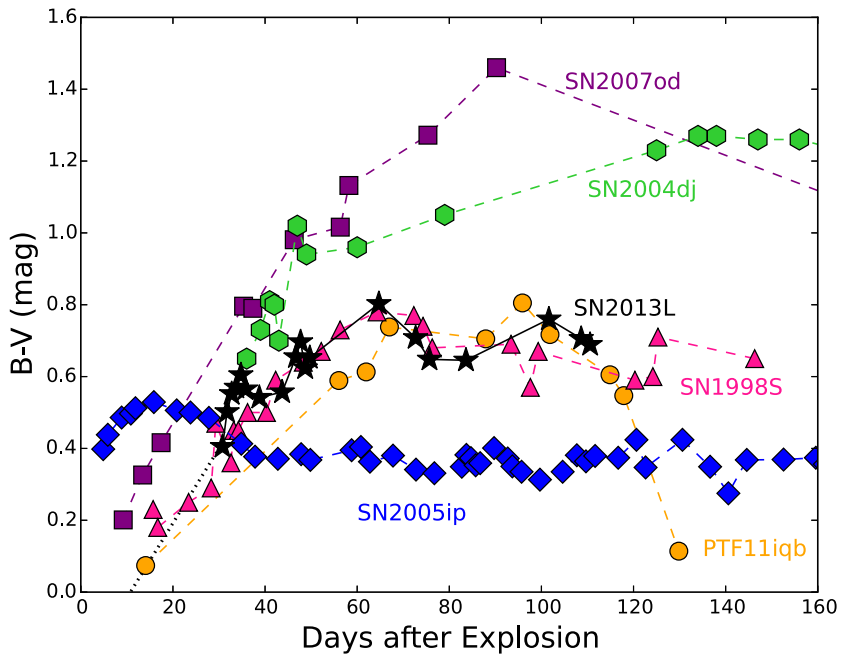

Figure 6. $B-V$ colour evolution of SN 2013L (black stars) compared with other CSM interacting IIn and IIP SNe. The dashed black line indicates a projected colour using the blackbody temperature of SN 2013L on day 5. Data are from Stritzinger et al. (2012, SN2005ip), Smith et al. (2015, PTF11iqb), Fassia et al. (2000, SN1998S), Inserra et al. (2011, SN2007od) and Zhang et al. (2006, SN2004dj). All data have been corrected for extinction using the $E(B-V)$ quoted in the corresponding papers, and using a standard reddening law of $\mathrm{R}_{v}$ (Cardelli et al. 1989).

\section{SPECTROSCOPIC EVOLUTION}

The first spectrum of SN2013L on day 5 after discovery, shown in grey at the bottom of Fig. 4, shows a mostly featureless continuum with only narrow hydrogen Balmer lines present in emission. A blackbody fit yields a temperature of $12000 \mathrm{~K}$ and a photospheric radius of $7 \times 10^{14} \mathrm{~cm}$, similar to other Type IIn $\mathrm{SNe}$ at around 


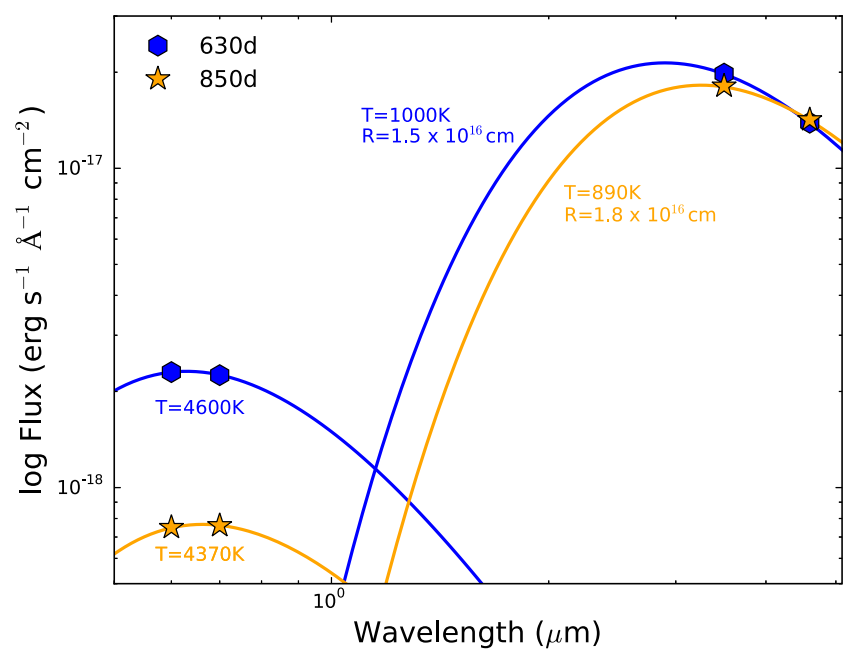

Figure 7. SED of SN2013L on day 630 and 850 . The IR points are from the Spitzer observations, while the optical data are the continuum emission on either side of $\mathrm{H} \alpha$ extrapolated from the day 482, 728, and 1133 optical spectra. The solid lines are the blackbody fits to both the optical and IR components of each epoch, and the corresponding temperatures and blackbody radius are indicated on the plot.

the same age (Smith et al. 2015; Kangas et al. 2016). There were no WR lines seen in the earliest spectrum, like was observed for other IIn SNe 1998S (Shivvers et al. 2015), SN 2013cu (Gal-Yam et al. 2014) and PTF11iqb (Smith et al. 2015). This does not, of course, discount their presence at earlier epochs.

Narrow P-Cygni absorption and emission of the Balmer lines and He I $10830 \AA$ can be seen in the spectrum on day 33. Narrow emission was also present on day 5 , but the low resolution of that spectrum makes the detection of an absorption feature impossible. In all but the $\mathrm{H} \alpha$ line, the absorption component is stronger than the emission. Narrow emission is also seen in the Paschen and Brackett series in the NIR, with no evidence of an absorption component. Similarly, the He I lines in the optical only show narrow emission.

The Balmer and $\mathrm{He}_{\mathrm{I}} 10830 \AA$ lines (with the addition of $\mathrm{Pa} \beta$ ) also show intermediate-width components with - full width at halfmaximum (FWHM) of $2000-4000 \mathrm{~km} \mathrm{~s}^{-1}$, with one centred at roughly zero velocity and the other centred a few thousand $\mathrm{km} \mathrm{s}^{-1}$ to the blue (Figs 8 and 9). A comparable red emission feature is not seen. We can fit the $\mathrm{H} \alpha$ profiles with three distinct features: a narrow emission profile with prominent P-Cygni absorption, an intermediate-width Lorentzian centred near zero velocity and an intermediate width Gaussian profile centred at roughly $-3500 \mathrm{~km} \mathrm{~s}^{-1}$. The broad Lorentzian profile occurs when optically thick material causes multiple electron-scattering events. The resulting narrow part of the profile traces the expansion velocity of the emitting material, in this case the slow moving progenitor wind or the CSM, while the broad wings are a function of the optical depth of the material (Chugai 2001). Fig. 10 shows the two intermediate-width components needed to create the multipeaked profile of $\mathrm{H} \alpha$ on day 109. Both the Lorentzian and the Gaussian components are blueshifted from the central narrow component. The FWHM and other information of the components are listed in Table 4 and shown in Fig. 11.

With time, the FWHM of the blueshifted component decreases, from roughly $3900 \mathrm{~km} \mathrm{~s}^{-1}$ on day 33 to $\sim 1100 \mathrm{~km} \mathrm{~s}^{-1}$ on our last observation on day 1509. During this same time period, the centre of the blue component shifts from -4000 to $-1800 \mathrm{~km} \mathrm{~s}^{-1}$. The behaviour of the central Lorentzian emission is a bit less
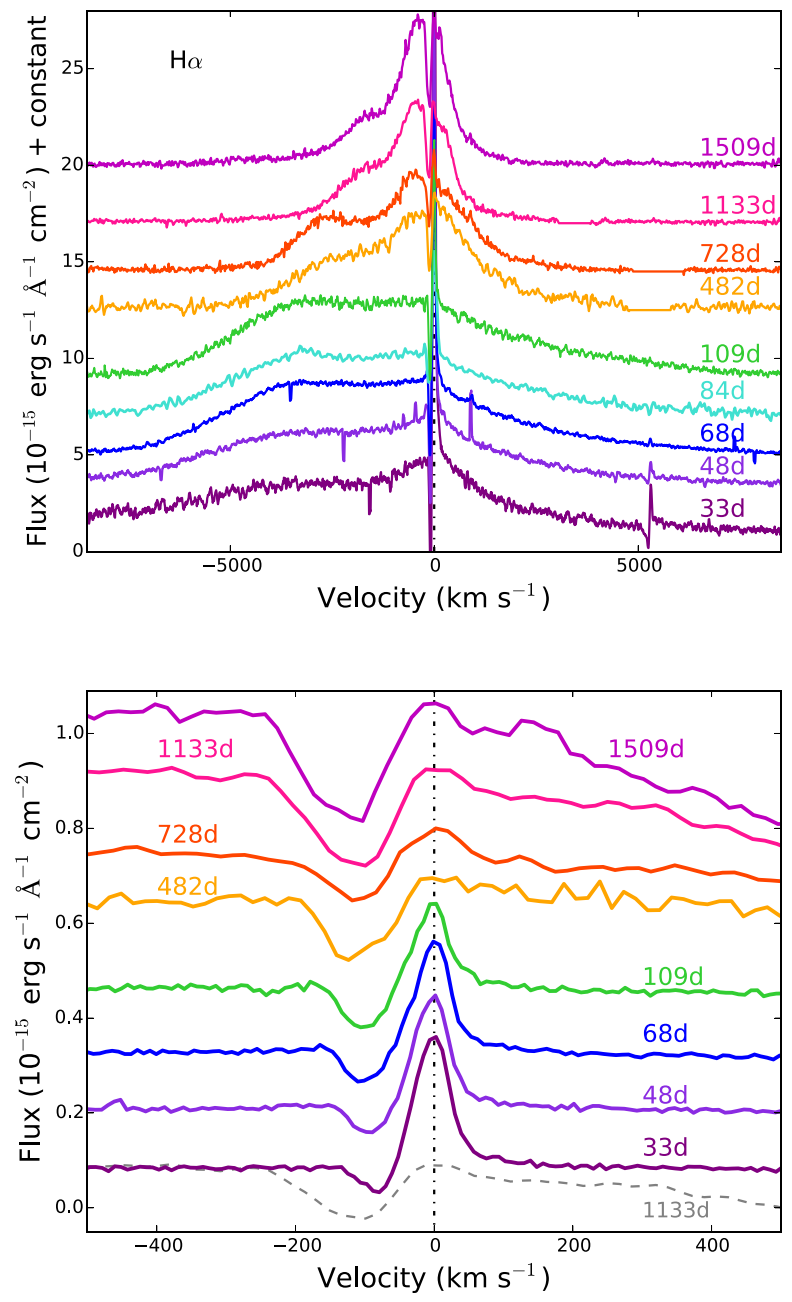

Figure 8. Spectral evolution of $\mathrm{H} \alpha$ in velocity space. The top panel shows the intermediate width emission lines and the evolution of the blue-bump with time, while the bottom panel is zoomed to show the evolution of the narrow emission and absorption components. The comparison between the narrow line shape on day 33 and 1133 can be seen in the bottom panel as the dashed grey line is the emission from day 1133 scaled and shifted to match the blue side of the line. Zero velocity is indicated by the black dashdotted line, and has been chosen to be centred on the narrow $\mathrm{H} \alpha$ emission.

Table 4. FWHM and centres of $\mathrm{H} \alpha$ Components in $\mathrm{km} \mathrm{s}^{-1}$.

\begin{tabular}{lccccc}
\hline $\begin{array}{l}\text { Epoch } \\
\text { (days) }\end{array}$ & Narrow Abs & \multicolumn{2}{c}{ Lorentzian } & \multicolumn{2}{c}{ Gaussian } \\
$v_{\text {centre }}$ & FWHM & $v_{\text {centre }}$ & FWHM & $v_{\text {centre }}$ \\
\hline 33 & -84 & 2696 & -100 & 3869 & -3985 \\
48 & -91 & 4047 & -328 & 3640 & -3894 \\
68 & -100 & 4949 & -420 & 3275 & -3772 \\
109 & -98 & 5275 & -337 & 2935 & -3515 \\
482 & -104 & 2472 & -221 & 2039 & -2644 \\
728 & -114 & 2113 & -292 & 1495 & -2669 \\
1133 & -126 & 1484 & -253 & 1265 & -1808 \\
1509 & -130 & 1258 & -210 & 1084 & -1810 \\
\hline
\end{tabular}

straightforward, with the FWHM rising to a maximum of $\sim 5300 \mathrm{~km} \mathrm{~s}^{-1}$ on day 109 , and then gradually falling again to $\sim 1300 \mathrm{~km} \mathrm{~s}^{-1}$ by day 1509 . The narrow emission also gets broader and fainter, and by day 482, the narrow absorption component is more pronounced and is in fact stronger than the narrow emission 

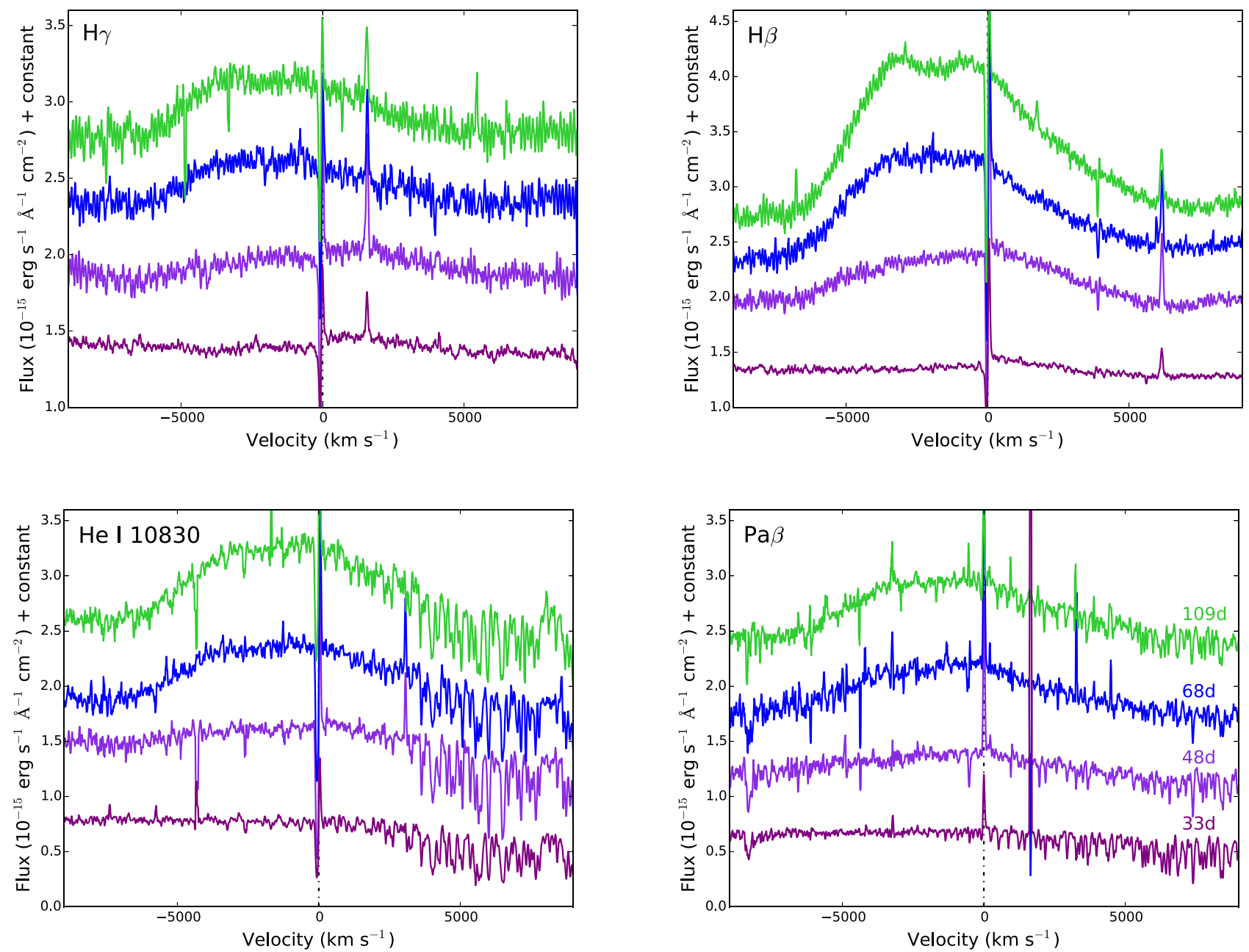

Figure 9. Same as Fig. 8, but in velocity space zoomed in on various hydrogen or helium emission lines which are indicated in the top corner of the plot. Only the epochs with the highest resolution are included in this plot, and all NIR emission is from only the XSHOOTER spectra. Zero velocity is indicated by a dashed vertical line.

by our last observations. The fact that we are still seeing narrow P-Cygni absorption even on our last observation on day 1509 may provide important clues about the density and geometry of the CSM as well as our viewing angle. Similar profiles have been seen in SN 1998S and PTF11iqb and will be discussed in detail below.

At no time do the [O I] lines at $\lambda \lambda 6300,6363 \AA$ appear, and there is a general lack of a nebular phase typically seen in normal Type IIP SNe. This is common in SNe IIn, where CSM interaction tends to mask the underlying nebular phase spectrum (see Smith 2014). There does appear to be an intermediate-width feature around $8500 \AA$ that was also seen in the spectrum of SN 2010j1 presented in Fransson et al. (2014). There it was attributed to O I $\lambda 8446 \AA$ emission, but as we show in Fig. 10, we can approximate the shape of the emission in SN 2013L by using the $\mathrm{H} \alpha$ profile of the same time centred at each of the Ca II IR triplet locations $(\lambda \lambda \lambda 8498,8542,8662 \AA)$. While not an exact fit, the combination of self-absorption and broad line-widths can account for the discrepancy. No narrow lines are seen at any of the Ca II IR locations, suggesting that $\mathrm{Ca}$ II emission lines arise in the fast ejecta or at the location of the reverse shock.

In our last epoch on day 1509, the narrow emission seems to have all but vanished, and only the narrow absorption feature remains. Assuming that slow RSG winds reach as far as $3 \times 10^{18} \mathrm{~cm}$, or
3.2 ly (Lloyd, O'Brien \& Kahn 1995), we would expect the narrow emission lines ionized by the SN blast wave to begin fading by $\sim 3 \mathrm{yr}$. The persistence of the narrow absorption may be an indication of geometry; if there is an equatorial enhancement of the CSM, we may be viewing that nearly edge-on. Continued monitoring will reveal how that absorption feature evolves, and could potentially show the appearance of the [O I ] emission lines as the oxygen layers begin to interact with the reverse shock, as was seen in SN 1998S (Mauerhan \& Smith 2012).

\subsection{The multipeaked shape of $\mathrm{H} \alpha$}

One of the most striking features of SN 2013L is the multicomponent structure of $\mathrm{H} \alpha$ (and other Balmer and $\mathrm{He}_{\mathrm{I}}$ lines), in particular the additional blueshifted emission around $-3500 \mathrm{~km} \mathrm{~s}^{-1}$. Often, a blueshifted asymmetry can be explained by dust formation attenuating the receding red side of the SN more so than the blue. First detected in SN 1987A (Lucy et al. 1989), this type of evidence for dust formation has been seen in many CCSNe including SN 2003gd (Sugerman et al. 2006), SN 2004et (Kotak et al. 2009), SN 2005ip (Fox et al. 2010; Smith et al. 2011b), SN 2007od (Andrews et al. 2010) and one of the clearest cases SN 2006jc (Smith, Foley \& Filippenko 2008a). In conjunction with the emission line 

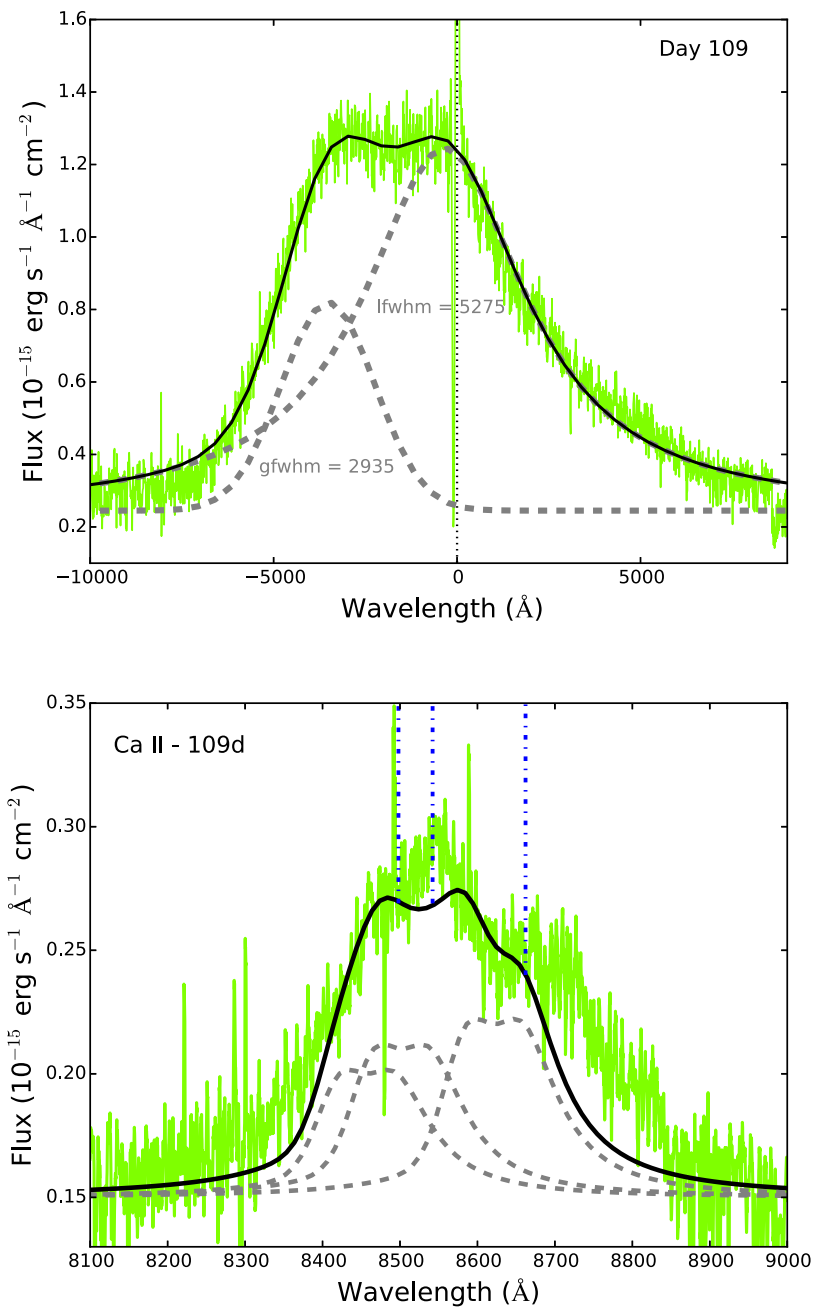

Figure 10. Top: Multicomponent fit (black line) to the broad $\mathrm{H} \alpha$ emission line on day 109 (green). Allowing the narrow emission to reside at zero velocity, the profile can be recreated with a Lorentzian centred at $-337 \mathrm{~km} \mathrm{~s}^{-1}$ and a Gaussian blueshifted to $\sim 3515 \mathrm{~km} \mathrm{~s}^{-1}$, both of which are shown by the dashed grey line. Bottom: The Ca II $\lambda \lambda \lambda 8498,8542,8662 \AA$ IR triplet from day 109 (green) with a fit created from scaling and combining the fit from the $\mathrm{H} \alpha$ profile seen above for each of the three lines. Each individual profile is shown in the dashed grey line.

asymmetry, a decrease in the optical light curve and a corresponding increase in the IR brightness is often observed as new dust grain form in the ejecta. No early IR data exist for SN 2013L as we mention above, and any optical obscuration in the light curve due to dust can be hidden by the ongoing CSM interaction. The lack of any emission on the red side of $\mathrm{H} \alpha$ in all epochs, plus the presence of the blue emission by day 33 when ejecta temperatures would probably have been too high for dust condensation may suggest another mechanism for the multipeaked lines.

In Fig. 12, we plot $\mathrm{H} \alpha$ and $\mathrm{Pa} \beta$ from our day 109 spectrum. Adjusting the flux of the $\mathrm{Pa} \beta$ emission line to match that of $\mathrm{H} \alpha$ shows that there is little difference in the profile shape. This further supports the hypothesis that extinction from newly formed dust is not the cause for the asymmetry. If it were due to dust, the red side of $\mathrm{H} \alpha$ would be more attenuated than that of $\mathrm{Pa} \beta$ due to the wavelength dependence of the dust extinction. Since the bluer $\mathrm{H} \alpha$ shows the same shape as the redder $\mathrm{Pa} \beta$, the asymmetry is intrinsic to the $\mathrm{SN}$ or the CSM.

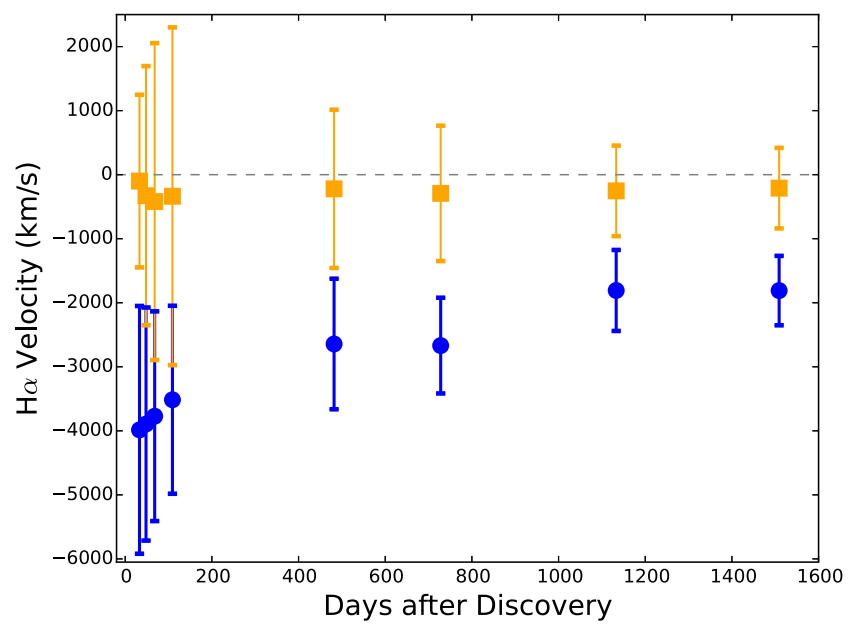

Figure 11. Evolution of the centroid and FWHM of the Lorentzian (orange) and Gaussian (blue) emission of $\mathrm{H} \alpha$ with time. The centroid is shown as the square or circle symbol, and the FWHM by the velocities spanned by the vertical bars.

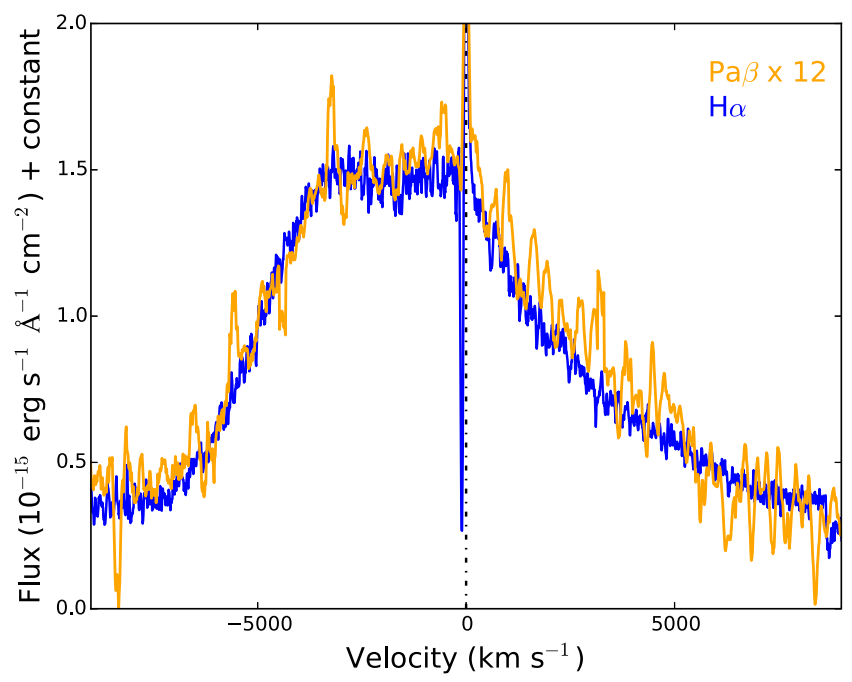

Figure 12. Comparison of $\mathrm{H} \alpha$ (blue) and $\mathrm{Pa} \beta$ (orange) emission on day 109 .

While we cannot rule out that there is some newly formed dust responsible for a portion of the hydrogen asymmetry, it is more likely an asymmetry in the surrounding CSM. If we invoke a disclike geometry of the CSM and then view it edge-on the CSM can easily obscure the red side of the emission. This is further supported by the persistent narrow P-Cygni lines, particularly the absorption feature at late times. On day 1509 , the blue emission bump is centred at $-1810 \mathrm{~km} \mathrm{~s}^{-1}$, this would mean the minimum radius for the location of the material responsible for the blue emission bump is $\sim 2 \times 10^{16} \mathrm{~cm}(\sim 1330 \mathrm{au})$ away. Of course instead of a disc seen edge-on and therefore obscuring the receding side, we could potentially have a situation with only CSM on the blue side of the $\mathrm{SN}$, similar to PTF11iqb that showed a density enhancement on the red side (Smith et al. 2015). The one-sided geometry (i.e. the blue emission bump at $-3500 \mathrm{~km} \mathrm{~s}^{-1}$ ) could be explained by an SN exploding in a binary system where mass transfer is occurring preferentially to one side due to orbital eccentricities. Case in point is RY Scuti that is in a state-of-mass transfer from a binary companion and shows a resolved toroidal CSM with a density enhancement on 


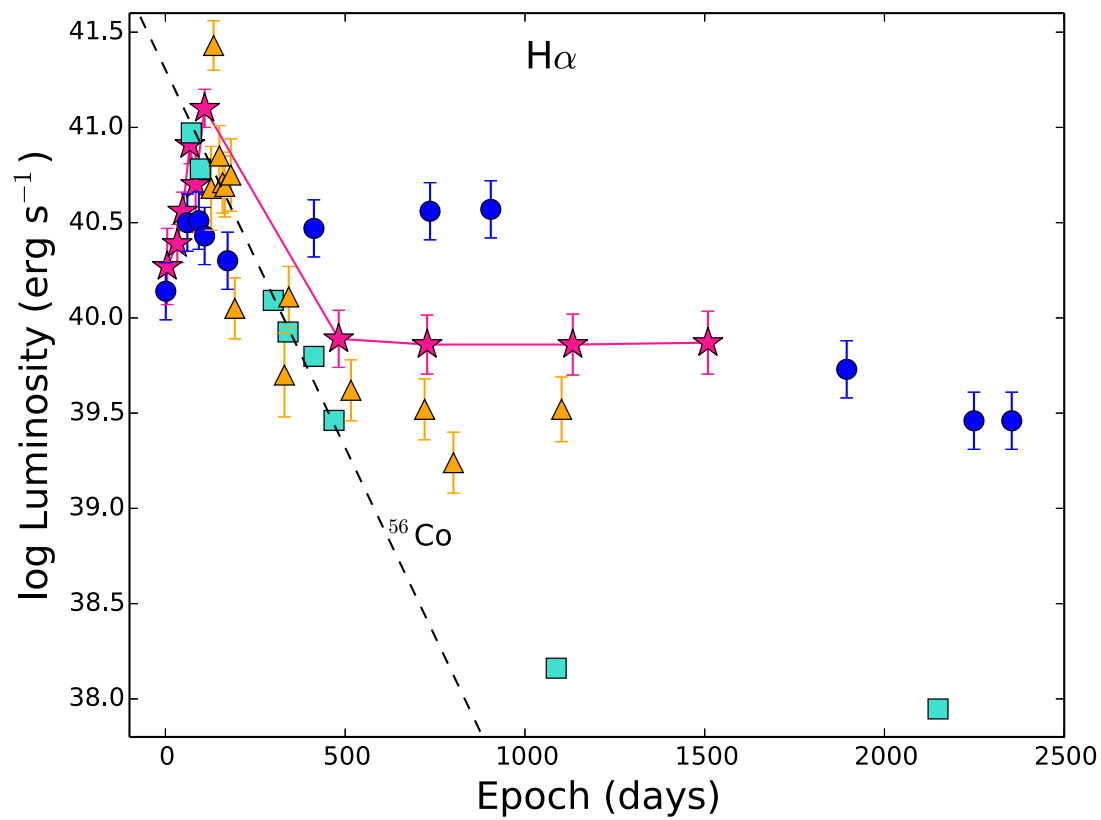

Figure 13. $\mathrm{H} \alpha$ luminosity of SN2013L (pink stars) compared with other IIn SNe. Data for 1998S (teal squares) come from fig. 4 of Mauerhan \& Smith (2012), for PTF11iqb (orange triangles) from fig. 9 of Smith et al. (2015), and for SN2005ip (blue circles) from Smith et al. (2017). The radioactive decay luminosity of ${ }^{56} \mathrm{Co}$ is plotted as a dashed black line.

the far side (Gehrz et al. 2001; Smith et al. 2002, 2011d). A onesided eruption is another possibility to create an asymmetric event. For instance Kiminki, Reiter \& Smith (2016) show that $\eta$ Carinae experience asymmetric, one-sided eruptions in 1250 and $1550 \mathrm{AD}$, centuries prior to the Great Eruption in the 1800s.

Asymmetric CSM is not uncommon, and over the past decade as the number of well-observed $\mathrm{CCSNe}$ has grown, many $\mathrm{SNe}$ IIn have displayed evidence of disc, torus or bipolar CSM. This includes SN 1998S (Leonard et al. 2000), SN 1997eg (Hoffman et al. 2008), SN 2009ip (Levesque et al. 2014; Mauerhan et al. 2014; Smith, Mauerhan \& Prieto 2014), SN 2010jl (Andrews et al. 2011; Patat et al. 2011; Smith et al. 2012; Chandra et al. 2015; Katsuda et al. 2016), PTF11iqb (Smith et al. 2015) and SN 2015bh (Elias-Rosa et al. 2016). Recently, Mauerhan et al. (2017) suggested that the IIP/L SN2013ej SN likely had an equatorial enhanced CSM close to edge on based on spectropolarimetry. Similarly, SN 2004dj (Chugai 2006), SN 2007od (Andrews et al. 2010), SN2011ja (Andrews et al. 2016) and other type IIP/L also show observational signatures of asymmetric CSM (Fig. 14). While not exhibiting the narrow-line emission, the late-time $\mathrm{H} \alpha$ of these IIP/L SNe was multipeaked, suggesting that a continuum of CSM interaction strengths and geometries exists. SN 2013L may be similar to those, but it also happens to have closer and denser CSM that made it appear as a Type IIn.

\section{DISCUSSION}

\subsection{The enduring luminosity of $\mathrm{H} \alpha$}

The late-time optical emission of SN 2013L is powered by the SN ejecta colliding with the CSM, which is clearly illustrated in the most recent spectroscopic observations. From day 482 and onwards, the optical spectra are dominated by strong $\mathrm{H} \alpha$ emission. To illustrate the strength of this late-time emission, in Fig. 13 we have plotted the total integrated $\mathrm{H} \alpha$ luminosity of SN 2013L from each of the epochs listed in Table 3, along with other well studied and enduring IIn SNe. This figure is adapted from Smith et al. (2017) and Mauerhan \& Smith (2012). SN 2013L starts with $L_{\mathrm{H} \alpha}$ similar to that of SN 2005ip, but quickly rises to the levels of PTF11iqb. The emergence of the blue bump in the Balmer lines by day 33 and the plateau in the $R$-band light curve after day $\sim 50$ suggest that the ejecta began interacting with even denser CSM from the very start of explosion. There are no spectra available between days 109 and 482 , so it is possible the luminosity continued to rise before declining down to the level detected over a year later, or as is suggested by Fig. 13, it could have declined at roughly the radioactive ${ }^{56} \mathrm{Co}$ decay rate.

By day 482 , the $L_{\mathrm{H} \alpha}$ is much brighter than would be expected from radioactivity alone and remains at roughly this brightness even on our last observation on day 1509 . This is evidence of interaction powered $\mathrm{H} \alpha$. On day $1133, \mathrm{SN} 2013 \mathrm{~L}$ is $\sim 45 \times$ brighter than SN 1998S at the same age, and $\sim 2.5 \times$ brighter than PTF11iqb. There is a lack of data for SN 2005ip during this time period, but we can estimate SN 2013L was likely $\sim 2-3 \times$ fainter. This would suggest that SN2013L has a wind density roughly halfway between SN 2005ip and PTF11iqb, or a mass-loss rate between $1 \times 10^{-4}$ and $1 \times 10^{-3} \mathrm{M}_{\odot} \mathrm{yr}^{-1}$ (Smith et al. 2009a, 2015, 2017). Further comparisons among these three objects will be explored below.

Although we do not have X-ray or radio data of SN 2013L, we can use the luminosity and kinematics of the system offered to us through our optical spectra to determine a wind-density parameter and therefore a mass-loss rate of the progenitor star. This is expressed as $\frac{\dot{M}}{V_{\mathrm{CSM}}}=\frac{2 L}{V_{\mathrm{SN}}^{3}}$, where $V_{\mathrm{CSM}}$ is the CSM velocity measured from the minimum of the narrow P-Cygni lines, $V_{\mathrm{SN}}$ is the supernova expansion velocity and $L=L_{\mathrm{H} \alpha} / \epsilon$ (see Smith 2016). As a lower bound we can expect $\epsilon \sim 50$ percent, since the thermal energy produced should be about half of the kinetic energy. For the upper bound, we assume a conservative $L_{\mathrm{H} \alpha}=0.1 L$. SN 2010j1, PTF11iqb and SN 2009ip may have similar CSM geometries and efficiency factors used for these objects are 5-7 percent 

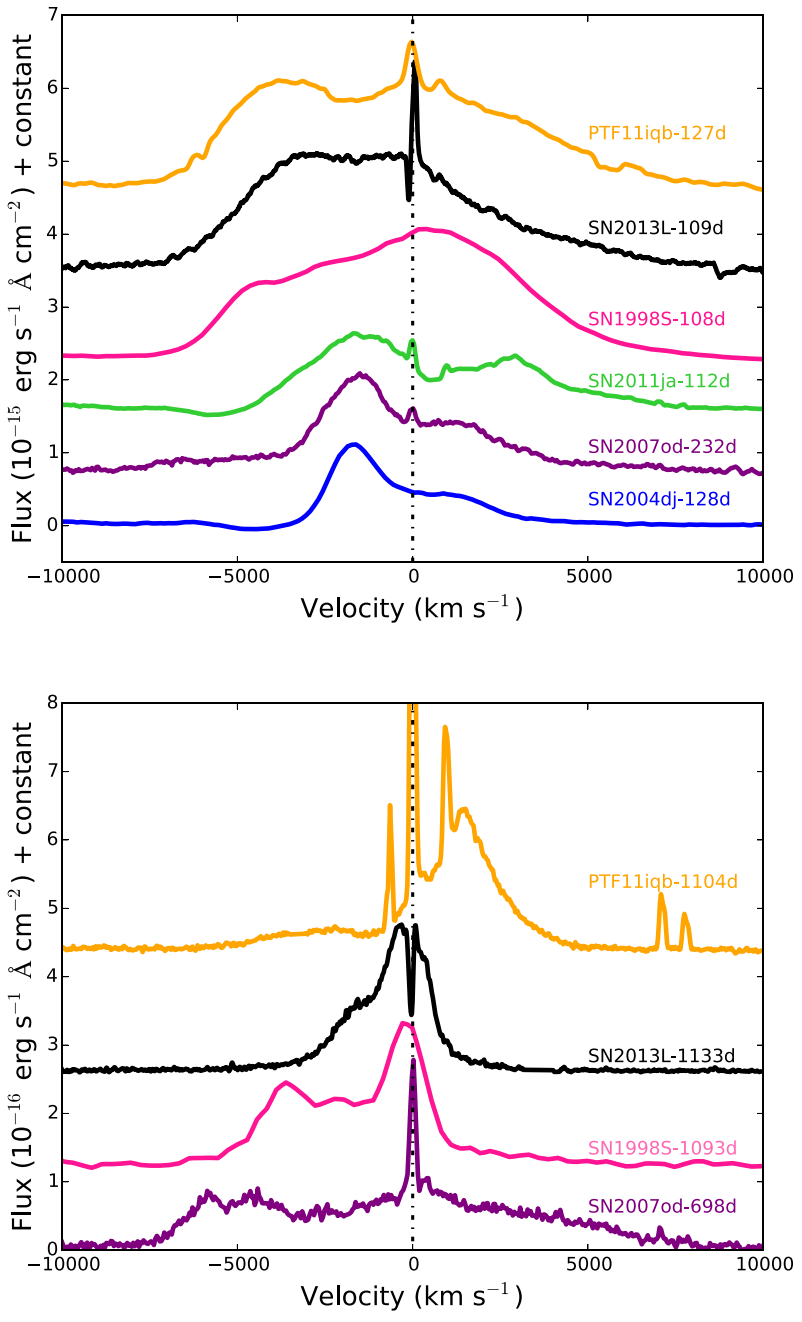

Figure 14. Comparison of the $\mathrm{H} \alpha$ profile of SN 2013L with other interacting CCSN at early (top) and late (bottom) times. Top: SN 2013L on day 109 in relation to three IIP SNe 2004dj (Vinkó et al. 2006), 2007od (Andrews et al. 2010) and 2011ja (Andrews et al. 2016) as well as two IIn SNe 1998S and PTF11iqb (Smith et al. 2015) at similar ages. Bottom: Same as top but for day 1133 for SN 2013L as well as much later times for SNe 2007od, 1998S and PTF11iqb.

(Fransson et al. 2014), 15 percent (Smith et al. 2015) and $<10$ percent (Mauerhan et al. 2014), respectively. On day 33, $V_{\mathrm{CSM}}=84 \mathrm{~km} \mathrm{~s}^{-1}, V_{\mathrm{SN}}=2696 \mathrm{~km} \mathrm{~s}^{-1}$ and $L_{\mathrm{H} \alpha}=10^{40.4}$, resulting in an $\dot{M}=0.34-1.75 \times 10^{-3} \mathrm{M}_{\odot} \mathrm{yr}^{-1}$. The same mass-loss rate is recovered within uncertainties throughout the evolution of the $\mathrm{SN}$, and by day 1509 has increased to $\sim 1.5-7.6 \times 10^{-3} \mathrm{M}_{\odot} \mathrm{yr}^{-1}$, consistent with the mass-loss rates of PTF11iqb and SN 2005ip as hypothesized above. Combined with wind velocities between 80 and $130 \mathrm{~km} \mathrm{~s}^{-1}$ this points to either a YHG or a non-eruptive LBV progenitor for SN 2013L (Kiewe et al. 2012; Smith 2016).

\subsection{Progenitor characteristics}

As reported in Table 3, the resolving power of the XSHOOTER observations is $\sim 7400$ or $40 \mathrm{~km} \mathrm{~s}^{-1}$. The measured P-Cygni absorption of the narrow emission component is $\sim 100 \mathrm{~km} \mathrm{~s}^{-1}$, larger than the resolution of the data. Therefore, we can assume that we are resolving the line and that the CSM surrounding the $\mathrm{SN}$ has a wind speed of $80-130 \mathrm{~km} \mathrm{~s}^{-1}$ depending on the epoch. This wind velocity places the progenitor in a YHG or LBV wind regime that suggests a $\mathrm{M}_{\text {ZAMS }}>25 \mathrm{M}_{\odot}$.

As has been eluded to throughout the text, the $\mathrm{H} \alpha$ profile seems to suggest disc-like geometry. Rings and discs indicating asymmetric mass-loss (perhaps due to interaction with a binary companion) are common around many evolved massive stars (Brandner et al. 1997; Smith, Bally \& Walawender 2007b; Wallström et al. 2017). The high instances of massive stars in binaries, of which 70-75 per cent will likely interact (Sana et al. 2012; Kiminki \& Kobulnicky 2012; de Mink et al. 2014), make a binary system that causes equatorial mass-loss likely. Close-binary progenitors are often invoked for Type IIb SNe (Podsiadlowski et al. 1993; Maund et al. 2011; Smith et al. 2011b; Benvenuto, Bersten \& Nomoto 2013; Fox et al. 2014), wherein the very nearby companion strips off the hydrogen envelope and the SN explodes as a YSG with only trace amounts of $\mathrm{H}$ in the atmosphere. If instead the binaries are further apart the radius of the RSG can increase as nuclear burning progresses, causing mass-loss and binary interaction (Smith \& Arnett 2014). This mass-loss would most likely be disc like and reside in the equatorial plane.

The high mass-loss rate, the asymmetric $\mathrm{H}$ lines, and the persistent narrow absorption lines are all suggestive of a disc or torus of CSM residing close the progenitor of SN 2013L that was created due to a binary companion. The fact that we see a IIn and not a Ib/c or IIb would seem to indicate that system was in RLOF when the progenitor exploded since far too much hydrogen is still present for substantial atmospheric stripping to have occurred. The progenitor scenario for SN 2013L could even be similar to the current YHG star HR 5171A. This highly evolved star appears to be in Wind RocheLobe Overflow with a much smaller companion, wherein the YHG wind does not completely fill the RL, but it is still gravitationally stripped on to the companion (Chesneau et al. 2014).

\subsection{Comparisons with other IIn}

\subsubsection{SNe 1998S and PTF11iqb}

The unique profiles and persistent luminosity of $\mathrm{H} \alpha$ in SN 2013L warrant a comparison with other CCSNe that show signs of strong CSM interaction. As is clearly seen in Fig. 5, with an absolute magnitude of -19.0 , SN 2013L is on par with PTF11iqb (-18.4) and SN 1998S (-19.5). Not only are the absolute magnitudes similar, but the $B-V$ colour evolution (Fig. 6) of SN 2013L, SN 1998S and PTF11iqb are also almost identical in the first few months. Around $100 \mathrm{~d}$, the light curves begin to diverge, with PTF11iqb and SN 1998S continuing to drop in magnitude (the former at a less drastic rate than the later) and SN 2013L hitting a plateau in $R$-band brightness. From these two diagnostics alone, it would seem that these three objects belong to their own subset of IIn CCSNe, but the addition of the $\mathrm{H} \alpha$ profiles further support this hypothesis.

Illustrated in Fig. 14, the multipeaked $\mathrm{H} \alpha$ seen in SN 2013L is reminiscent of that seen in SN 1998S and PTF11iqb. While the complex $\mathrm{H} \alpha$ was seen starting by day 33 in SN 2013L and day 37 in PTF11iqb, it was not until $\sim 200 \mathrm{~d}$ when the multiple peaks appeared in SN 1998S (although asymmetries were present by day 100). Unlike PTF11iqb that shifts from having a prominent blue peak early on, to a red peak in $\mathrm{H} \alpha$ after day 500, SN 2013L has a persistent blue asymmetry until our last spectra on day 1509. SN 1998S also maintains a blue peak well past $\sim 1000$ d. Elsewhere in the optical spectra the similarities end. Unlike PTF11iqb and SN 1998S, SN 2013L does not show nebular emission lines in the 
spectra except for the CaII IR triplet, supporting the presence of strong CSM interaction that masks the ejecta emission.

For comparison we have added other non-IIn CCSNe SN 2004dj, SN 2007od and SN 2011 ja, which also show asymmetric and multipeaked $\mathrm{H} \alpha$, and have compared the profiles both at early $(\sim 100 \mathrm{~d})$ and late $(\sim 1000 \mathrm{~d})$ times. Obvious in the early-time comparison is the lower velocities of the blue component of the non-IIn objects. SN 2004dj exhibited a blue peak at $-1770 \mathrm{~km} \mathrm{~s}^{-1}$ (Vinkó et al. 2006; Meikle et al. 2011), SN 2007od showed a blue peak at roughly $-1500 \mathrm{~km} \mathrm{~s}^{-1}$ (Andrews et al. 2010) and SN 2011ja at $-1400 \mathrm{~km} \mathrm{~s}^{-1}$ (Andrews et al. 2016). The IIn SNe, however, have blue peaks residing between -3500 and $-5000 \mathrm{~km} \mathrm{~s}^{-1}$. There is a $-5000 \mathrm{~km} \mathrm{~s}^{-1}$ peak that begins to emerge at late times in $\mathrm{SN}$ 2007od, likely indicative of CSM/ejecta interaction much further from the explosion, but it is a separate component from the early peak at $-1500 \mathrm{~km} \mathrm{~s}^{-1}$

The measured CSM velocities for PTF11iqb, SN 1998S and SN 2013L are similar and indicative of a high-mass-extended progenitor, with 1998S exhibiting $40 \mathrm{~km} \mathrm{~s}^{-1}$ speeds (Shivvers et al. 2015), PTF11iqb has a resolution-limited CSM velocity of $<80 \mathrm{~km} \mathrm{~s}^{-1}$ (Smith et al. 2015) and SN 2013L at $84 \mathrm{~km} \mathrm{~s}^{-1}$. The mass-loss rates are also similar, with an $\dot{M} \sim 0.3-7.6 \times 10^{-3} \mathrm{M}_{\odot} \mathrm{yr}^{-1}$ of SN $2013 \mathrm{~L}$ falling comfortably with both PTF11iqb $\left(\dot{M} \sim 1 \times 10^{-4} \mathrm{M}_{\odot} \mathrm{yr}^{-1}\right.$; Smith et al. 2015) and SN 1998S $\left(\dot{M} \sim 2 \times 10^{-5} \odot \mathrm{yr}^{-1}\right.$; Fassia et al. 2001), albeit on the higher side. While both PTF11iqb and SN 1998S have mass-loss rates and progenitor wind speeds that are suggestive of extreme RSG, YSG or YHG progenitors, the slightly elevated wind speed and $\dot{M}$ of SN 2013L may be more likely due to a YHG or LBV progenitor.

\subsubsection{SN 2005ip}

It is also worth taking a moment to discuss how SN 2013L is like and unlike the well-studied IIn SN 2005ip. While the $\mathrm{H} \alpha$ luminosity of both objects began roughly equally, SN 2005ip is about $5 \times$ more luminous (Fig. 13) than SN 2013L at day $\sim 730$. The $R$-band light curve behaves similarly, but with the maximum absolute magnitude of SN 2013L reaching roughly a magnitude brighter (Fig. 5) before fading below that of SN $2005 i p$ after $500 \mathrm{~d}$. Additionally, the colour evolution over the first $100 \mathrm{~d}$ is quite different, with SN 2005ip staying much bluer at a constant $(B-V)=0.40$, while SN 2013L was gradually becoming redder (Fig. 6). Nebular and coronal lines were prominent in SN 2005ip, but not seen in SN 2013L. These differences could be attributed to asymmetries, as SN 2005ip seems to be mostly spherical unlike the disc-like shape inferred for $\mathrm{SN}$ 2013L. This could account for the much brighter $\mathrm{H} \alpha$ luminosity of SN 2005ip that has a much larger area of CSM that can interact with the SN ejecta.

\section{CONCLUSIONS}

SN 2013L is an enduring SN IIn with long-lasting CSM interaction and prominent multipeaked $\mathrm{H} \alpha$ emission. The multiple components arise from the geometry of the system and the mass-loss history of the SN progenitor. Narrow emission comes from the ionization of the slow-moving wind from the progenitor, intermediate-width components from the ejecta/CSM interaction and underlying broad emission from the freely expanding ejecta. The blueshifted hydrogen component that is seen in all of our epochs, and the lack of a redshifted component, indicates that either the CSM material is distributed asymmetrically between us and the $\mathrm{SN}$ or that there is a disc or torus of CSM. The narrow absorption that persists until the last observation on day 1509 suggests that we are seeing the system from roughly edge on. The onset of CSM interaction by day 5 and persisting until our last observation on day 1509 suggests a dense and/or very extended CSM. If this object behaves anything like SNe 2005ip and $1988 \mathrm{Z}$ we may continue seeing signs of CSM interaction for years to come.

The narrow P-Cygni absorption indicates an outflow wind velocity between 80 and $130 \mathrm{~km} \mathrm{~s}^{-1}$, speeds commonly associated with a YHG or quiescent LBV wind (Smith 2014). The observations we present here suggest that SN 2013L has a more asymmetric and less inclined CSM than the similar IIn SNe 1998S or PTF11iqb, and were it to have a spherically distributed CSM would closely resemble SN 2005ip. While the absolute magnitude of SN 2013L does not put it in the superluminous $\mathrm{SN}$ regime, it is bright enough to require an extreme amount of mass-loss in the years prior to explosion. With objects such as SN 2013L and PTF11iqb, it becomes more apparent that there are multiple progenitor scenarios that can likely give rise to early and strong CSM interaction, including RSGs in a binary system. The addition of CSM asymmetry in Type IIn SNe allows us to be able to create a continuum among the non-super luminous IIn, and opens the door for RSGs to be common progenitors for all but the brightest SNe IIn.

\section{ACKNOWLEDGEMENTS}

Special thanks to Melissa Graham for observations and data reduction of SN 2013L taken with LCO and to the anonymous referee for constructive comments and suggestions. This research has made use of the services of the ESO Science Archive Facility and includes data gathered with the $6.5 \mathrm{~m}$ Magellan Telescopes located at Las Campanas Observatory, Chile. This work makes use of observations from the LCO network and is based in part on observations from Spitzer Space Telescope. Support was provided by the National Science Foundation (NSF) through grants AST-1210599 and AST-1312221 to the University of Arizona. This research has made use of the NED that is operated by the Jet Propulsion Laboratory, California Institute of Technology, under contract with the National Aeronautics and Space Administration.

\section{REFERENCES}

Andrews et al., 2010, ApJ, 715, 541

Andrews J. E. et al., 2011, AJ, 142, 45

Andrews J. E. et al., 2016, MNRAS, 457, 3241

Aretxaga I., Benetti S., Terlevich R. J., Fabian A. C., Cappellaro E., Turatto M., della Valle M., 1999, MNRAS, 309, 343

Ballesteros F. J., 2012, EL, 97, 34008

Benetti S., Cappellaro E., Danziger I. J., Turatto M., Patat F., della Valle M., 1998, MNRAS, 294, 448

Benvenuto O. G., Bersten M. C., Nomoto K., 2013, ApJ, 762, 74

Bilinski C., Smith N., Li W., Williams G. G., Zheng W., Filippenko A. V., 2015, MNRAS, 450, 246

Bonvin V. et al., 2017, MNRAS, 465, 4914

Brandner W., Grebel E. K., Chu Y.-H., Weis K., 1997, ApJ, 475, L45

Brown T. M. et al., 2013, PASP, 125, 1031

Cardelli J. A., Clayton G. C., Mathis J. S., 1989, ApJ, 345, 245

Chandra P., Chevalier R. A., Chugai N., Fransson C., Soderberg A. M., 2015, ApJ, 810, 32

Chesneau O. et al., 2014, A\&A, 563, A71

Chugai N. N., 2001, MNRAS, 326, 1448

Chugai N. N., 2006, Astron. Lett., 32, 739

de Mink S. E., Sana H., Langer N., Izzard R. G., Schneider F. R. N., 2014, ApJ, 782, 7

Dressler A. et al., 2011, PASP, 123, 288 
Elias-Rosa N. et al., 2016, MNRAS, 463, 3894

Fassia A. et al., 2000, MNRAS, 318, 1093

Fassia A. et al., 2001, MNRAS, 325, 907

Fox O. et al., 2009, ApJ, 691, 650

Fox O. D., Chevalier R. A., Dwek E., Skrutskie M. F., Sugerman B. E. K., Leisenring J. M., 2010, ApJ, 725, 1768

Fox O. D. et al., 2011, ApJ, 741, 7

Fox O. D., Filippenko A. V., Skrutskie M. F., Silverman J. M., Ganeshalingam M., Cenko S. B., Clubb K. I., 2013, AJ, 146, 2

Fox O. D. et al., 2014, ApJ, 790, 17

Fox O. D. et al., 2015, MNRAS, 454, 4366

Fox O. D. et al., 2017, ApJ, 836, 222

Fransson C. et al., 2014, ApJ, 797, 118

Gal-Yam A., Leonard D. C., 2009, Nature, 458, 865

Gal-Yam A. et al., 2014, Nature, 509, 471

Gall C. et al., 2014, Nature, 511, 326

Gehrz R. D., Smith N., Jones B., Puetter R., Yahil A., 2001, ApJ, 559, 395

Habergham S. M., Anderson J. P., James P. A., Lyman J. D., 2014, MNRAS, 441,2230

Hoffman J. L., Leonard D. C., Chornock R., Filippenko A. V., Barth A. J., Matheson T., 2008, ApJ, 688, 1186

Inserra C. et al., 2011, MNRAS, 417, 261

Jester S. et al., 2005, AJ, 130, 873

Kangas T. et al., 2016, MNRAS, 456, 323

Kangas T. et al., 2017, A\&A, 597, A92

Katsuda S. et al., 2016, ApJ, 832, 194

Kiewe M. et al., 2012, ApJ, 744, 10

Kiminki D. C., Kobulnicky H. A., 2012, ApJ, 751, 4

Kiminki M. M., Reiter M., Smith N., 2016, MNRAS, 463, 845

Kotak R. et al., 2009, ApJ, 704, 306

Leonard D. C., Filippenko A. V., Barth A. J., Matheson T., 2000, ApJ, 536, 239

Levesque E. M., Stringfellow G. S., Ginsburg A. G., Bally J., Keeney B. A., 2014, AJ, 147, 23

Lloyd H. M., O’Brien T. J., Kahn F. D., 1995, MNRAS, 273, L19

Lucy L. B., Danziger I. J., Gouiffes C., Bouchet P., 1989, LNP, 350, 164

Mauerhan J., Smith N., 2012, MNRAS, 424, 2659

Mauerhan J. C. et al., 2013a, MNRAS, 430, 1801

Mauerhan J. C. et al., 2013b, MNRAS, 431, 2599

Mauerhan J. et al., 2014, MNRAS, 442, 1166

Mauerhan J. C. et al., 2017, ApJ, 834, 118

Maund J. R. et al., 2011, ApJ, 739, L37

Meikle W. P. S. et al., 2011, ApJ, 732, 109

Monard L. A. G. et al., 2013, CBET, 3392, 1

Morales Garoffolo A. et al., 2013, Astron. Telegram, 4767, 1

Ofek E. O. et al., 2016, ApJ, 824, 6

Pastorello A. et al., 2002, MNRAS, 333, 27

Pastorello A. et al., 2013, ApJ, 767, 1
Patat F., Taubenberger S., Benetti S., Pastorello A., Harutyunyan A., 2011, A\&A, 527, L6

Podsiadlowski P., Hsu J. J. L., Joss P. C., Ross R. R., 1993, Nature, 364, 509

Quataert E., Shiode J., 2012, MNRAS, 423, L92

Sana H. et al., 2012, Sci, 337, 444

Schlegel E. M., 1990, MNRAS, 244, 269

Schlegel D. J., Finkbeiner D. P., Davis M., 1998, ApJ, 500, 525

Shivvers I., Groh J. H., Mauerhan J. C., Fox O. D., Leonard D. C., Filippenko A. V., 2015, ApJ, 806, 213

Smartt S. J. et al., 2015, A\&A, 579, A40

Smith N., 2014, ARA\&A, 52, 487

Smith N., 2016, preprint (arXiv:1612.02006)

Smith N., Arnett W. D., 2014, ApJ, 785, 82

Smith N., Gehrz R. D., Stahl O., Balick B., Kaufer A., 2002, ApJ, 578, 464

Smith N. et al., 2007a, ApJ, 666, 1116

Smith N., Bally J., Walawender J., 2007b, AJ, 134, 846

Smith N., Foley R. J., Filippenko A. V., 2008a, ApJ, 680, 568

Smith N., Chornock R., Li W., Ganeshalingam M., Silverman J. M., Foley R. J., Filippenko A. V., Barth A. J., 2008b, ApJ, 686, 467

Smith N. et al., 2009a, ApJ, 695, 1334

Smith N., Hinkle K. H., Ryde N., 2009b, AJ, 137, 3558

Smith N. et al., 2010, AJ, 139, 1451

Smith N. et al., 2011a, ApJ, 732, 63

Smith N., Li W., Filippenko A. V., Chornock R., 2011b, MNRAS, 412, 1522

Smith N., Li W., Silverman J. M., Ganeshalingam M., Filippenko A. V., 2011c, MNRAS, 415, 773

Smith N., Gehrz R. D., Campbell R., Kassis M., Le Mignant D., Kuluhiwa K., Filippenko A. V., 2011d, MNRAS, 418, 1959

Smith N., Silverman J. M., Filippenko A. V., Cooper M. C., Matheson T., Bian F., Weiner B. J., Comerford J. M., 2012, AJ, 143, 17

Smith N., Mauerhan J. C., Prieto J. L., 2014, MNRAS, 438, 1191

Smith N. et al., 2015, MNRAS, 449, 1876

Smith N. et al., 2017, MNRAS, 466, 3021

Stritzinger M. et al., 2012, ApJ, 756, 173

Sugerman B. E. K. et al., 2006, Science, 313, 196

Taddia F. et al., 2013, A\&A, 555, A10

Turatto M., Cappellaro E., Danziger I. J., Benetti S., Gouiffes C., della Valle M., 1993, MNRAS, 262, 128

Valenti S. et al., 2016, MNRAS, 459, 3939

Vinkó J. et al., 2006, MNRAS, 369, 1780

Wallström S. H. J. et al., 2017, A\&A, 597, A99

Yaron O., Gal-Yam A., 2012, PASP, 124, 668

Zhang T., Wang X., Li W., Zhou X., Ma J., Jiang Z., Chen J., 2006, AJ, 131, 2245

Zhang T. et al., 2012, AJ, 144, 131

This paper has been typeset from a $\mathrm{T}_{\mathrm{E}} \mathrm{X} / \mathrm{LT} \mathrm{E} \mathrm{X}$ file prepared by the author. 